\title{
Numerical investigation of nanoporous evaporation using direct simulation Monte Carlo
}

\author{
Benzi John $\odot,{ }^{1, *}$ Ryan Enright, ${ }^{2}$ James E. Sprittles $\odot,{ }^{3}$ Livio Gibelli, ${ }^{4}$ \\ David R. Emerson, ${ }^{1}$ and Duncan A. Lockerby ${ }^{5}$ \\ ${ }^{1}$ Scientific Computing Department, STFC Daresbury Laboratory, Warrington WA4 4AD, United Kingdom \\ ${ }^{2}$ Efficient Energy Transfer Department ( $\eta E T$ ), Nokia Bell Labs, Dublin D15 Y6NT, Ireland \\ ${ }^{3}$ Mathematics Institute, University of Warwick, Warwick CV4 7AL, United Kingdom \\ ${ }^{4}$ School of Engineering, University of Edinburgh, Edinburgh EH9 3FB, United Kingdom \\ ${ }^{5}$ School of Engineering, University of Warwick, Warwick CV4 7AL, United Kingdom
}

(Received 9 July 2019; published 4 November 2019)

\begin{abstract}
Evaporation is an effective cooling mechanism widely exploited in the thermal management of modern electronic devices, with a growing interest in the evaporation process in thin-film-based nanoporous membrane technologies. At such scales, classical approaches fail and one requires solutions of the Boltzmann equation; these are obtained here using the direct simulation Monte Carlo method. In particular, the evaporation from representative nanoporous meniscus shapes, corresponding to different operating conditions, has been investigated. Evaporation rates for the different conditions have been characterized as a function of a wide range of Knudsen numbers and free-stream Mach numbers. Additionally, the influence of porosity and evaporation coefficient on the nanoporous evaporation rates has been assessed. Investigations have also been carried out to consider cases where the meniscus has sunk within the pore, and cooling efficacy compared with cases where the meniscus is pinned to the top of the pore. This work demonstrates that the net evaporative mass flux is ultimately determined by the interplay between various physical effects, whose dominance is quantified by the Knudsen number, porosity, evaporation coefficient, and the meniscus shape. This work thus provides useful information for the design of nanoporous membrane-based cooling devices.
\end{abstract}

DOI: 10.1103/PhysRevFluids.4.113401

\section{INTRODUCTION}

Thermal management is becoming a prominent concern in the semiconductor industry [1-5] due to the ever-increasing power density in modern electronic devices. For instance, advanced electronics, such as gallium nitride high electron mobility transistors, generate heat fluxes exceeding $1000 \mathrm{~W} / \mathrm{cm}^{2}$. Traditional cooling techniques [6-10] like pool boiling, flow boiling, and jet impingement have been found to be inadequate for the thermal management of modern electronic devices, as they are only able to handle heat fluxes of the order $100 \mathrm{~W} / \mathrm{cm}^{2}$. Thin film evaporation [11] enabled by nanoporous membranes is a promising technology that has, in recent years, been proved to be capable of high heat-flux thermal management. The advantage of thin-film evaporation

\footnotetext{
*benzi.john@stfc.ac.uk
}

Published by the American Physical Society under the terms of the Creative Commons Attribution 4.0 International license. Further distribution of this work must maintain attribution to the author(s) and the published article's title, journal citation, and DOI. 
is that it minimizes the thermal resistance across the evaporating liquid and enhances the overall thermal transport, thereby making it possible to achieve a large critical heat flux. Additionally, as it relies on capillarity to passively supply the liquid, there is no requirement for significant pumping power to supply the liquid to the evaporating interface. Nano- and microwicks have been utilized [12-14] to generate capillary pressure and maintain a thin evaporating film. However, nano- and microwicks are also associated with large viscous losses, which can limit the overall performance of the thin-film evaporation-based device. Nanoporous membranes [15-18], on the other hand, not only generate high capillary pressure but also minimize the viscous losses as they are very thin, thereby reducing the flow transport length. Optimized semiconductor-based nanoporous membranes have thicknesses of $\sim 1 \mu \mathrm{m}$ and pore diameters of $L_{\mathrm{p}} \sim 100 \mathrm{~nm}$ (e.g., see [11,19]). Additionally, nanoporous membrane-based cooling devices are largely self-regulating in nature since they rely mainly on capillary pressure, $\Delta p \sim 2 \gamma R^{-1}$, mediated by surface tension, $\gamma$, and the interface radius of curvature, $R$, to drive the flow.

A critical understanding of the interfacial evaporation process at small scales is of interest not only from a fundamental perspective but also for the design of more efficient nanoporous membrane-based evaporative cooling devices. Additionally, evaporation from nanopores also has relevance to other applications like sea water desalination using carbon nanotubes [20,21], industrial separation processes [22], and natural processes like transpiration [23]. The key in all cases is to better understand the evaporation process that takes place at the liquid-vapor interface, so that the evaporative mass flux can be predicted accurately as a function of the operating conditions. Evaporation has been a well-researched topic with relevance to numerous industrial applications, and it is now widely recognized that gas kinetic aspects need to be accounted for when dealing with evaporation at the interface level, due to molecular nonequilibrium effects that occur near the interface, even at atmospheric pressure conditions [24].

Hertz and Knudsen are credited with pioneering work in this area, as they formulated an expression for evaporative mass flux, which is now well known as the classical Hertz-Knudsen formula $[25,26]$. However, the Hertz-Knudsen relation was derived without taking the macroscopic vapor velocity into account. Schrage [27] significantly improved this formula by including the vapor velocity, thereby including nonlinear convective effects. However, Schrage's equation assumes incorrectly that the entire fraction of molecules (having an equilibrium drifted Maxwellian distribution with a vapor velocity) adjacent to the Knudsen layer and moving towards the liquid phase condenses at the interface. Additionally, both the Hertz-Knudsen relation and Schrage's relation fail to accurately relate the external macroscopic flow (vapor) variables to the physical parameters at the interface. Subsequent works by other researchers, in particular, Labuntsov and Kryukov [28] and Ytrehus [24], overcame these limitations by formulating gas-kinetic moment equations of the Boltzmann equation. They conceived the interfacial evaporation process from a planar surface by essentially dividing the region of interest into a Knudsen layer near the interface (typically of the order of a few mean free paths thick, in which the vapor adapts to the given external equilibrium conditions) and an adjacent external hydrodynamic region with equilibrium vapor conditions. The coupling between the external flow variables and interface parameters was then achieved through numerical solutions of moments of the Boltzmann equation.

Other notable works on evaporation include detailed numerical solutions from nonlinear Boltzmann and Bhatnagar-Gross-Krook (BGK) equations given by Yen [29], Sone et al. [30], and Aoki et al. [31]. Numerical works based on the direct simulation Monte Carlo (DSMC) [32] method can be found in Murakami and Oshima [33] and Kogan and Abramov [34]. The results from kinetic theory for one-dimensional problems of evaporation and condensation at planar surfaces are considered to be fairly complete, particularly for monatomic gases. A comprehensive review on kinetic theoretical studies of the half-space problem of evaporation can be found in the work by Sone [35] and references therein. Studies on evaporation for polyatomic gases have been done by Cercignani [36] and Frezzotti [37,38]. Studies on evaporation from spheres [39,40] and from cylinders [41] have also been reported in the literature. 
The thermal performance of a nanoporous membrane evaporator is dependent on several aspects, including conduction heat transport to the liquid-vapor interface, liquid transport to the liquid-vapor interface, the vaporization process at the liquid-vapor interface and vapor transport from the interface. However, ultimately, the performance of a nanoporous membrane evaporator is governed by the evaporation kinetics at the liquid-vapor interface, which is where our research is focused. Regarding thin-film-based nanoporous membrane cooling, there have been several reported studies analyzing evaporation in nanopores. A recent experimental investigation, carrying out a parametric study on the effect of various geometric parameters on thin-film evaporation in nanoporous membranes, can be found in Wilke et al. [19]. Theoretical and numerical studies on nanoporous evaporation in the recent past $[11,18]$ essentially consider a one-dimensional resistance networktype model to couple the liquid phase flow with the interfacial process occurring at the liquid-vapor interface. Accordingly, the liquid flow was modeled using simplified versions of the Navier-Stokes equations, the interface was characterized with the augmented Young-Laplace equation [42], and the interfacial heat/mass flux was modeled using treatments from kinetic theory. The interfacial heat/mass flux in initial works was formulated using the Schrage equation. However, in later works the more accurate moment method solution of the Boltzmann equation, by Labuntsov and Kryukov [28], was used to account for nonequilibrium effects close to the liquid-vapor interface and to analyze evaporation from nanoporous membranes. The moment-based approach of Labuntsov and Kryukov has been shown to provide good agreement with numerical solutions of the Boltzmann transport equation $[43,44]$. However, it is to be noted that this approach has only been proved to be accurate and valid for evaporation from a planar surface, where the flow is one-dimensional. Its validity for significantly curved menisci cases under various operating conditions, where twodimensional effects may be important, needs to be further assessed. Additionally, the vapor flow within the nanopore needs to be treated accurately, particularly for the receding meniscus cases. Receding cases occur under severe operating conditions, such as in increased heat fluxes or liquid flow starvation, in which case the meniscus retreats down the pore.

A traditional continuum-based approach to evaluate the vapor flow within the nanopore is inadequate, as the pore size is comparable to the vapor mean free path. In previous works, this has been accounted for by modeling vapor flow using free molecular conditions within the pore and adopting a radiative thermal transport analogy approach [45], with free molecular transmission probabilities [46]. However, this approach will be inaccurate for significantly curved meniscus cases and lower Knudsen number conditions. Notably, a very recent study by Lu et al. [47] demonstrated both experimentally and numerically (using DSMC) that evaporation rates can be expressed as a function of the pressure ratio across the Knudsen layer. However, this study was limited to low Mach number cases and did not explore the crucial effect of meniscus shape or Knudsen number on the evaporation rates. Therefore, to summarize, although there have been attempts to study nanoporous evaporation, a detailed and fundamental understanding of this phenomenon as a function of various parameters like meniscus shape, Knudsen number, evaporation coefficient, etc., is lacking.

In the present study, a detailed numerical investigation of the evaporation process from the liquid-vapor interface of a representative nanopore is considered using the DSMC method. The gas dynamics and evaporation rates (mass flux) associated with different nanoporous evaporation conditions are the main focus here. Aspects like liquid-vapor interface structure, phase transition, effects of supersaturated vapor, etc. have not been covered in this study as these come under the realm of molecular dynamics simulations. Furthermore, liquid and sensible heat transport to the interface, or a coupling of the liquid with the vapor phase, have not been considered within the scope of this work. Instead, stationary fixed cases of menisci of different shapes and locations in the pore that are representative of different operating conditions in a nanoporous membrane evaporator are considered and their efficacies compared. In particular, we assess the evaporation rates as a function of a wide range of Knudsen numbers and free-stream Mach numbers and study the density ratio and temperature jump across the Knudsen layer. The impact of porosity on the evaporation rates is also assessed. The computed results are benchmarked against onedimensional analytical results from Labuntsov and Kryukov [28], wherever possible. Evaporation 


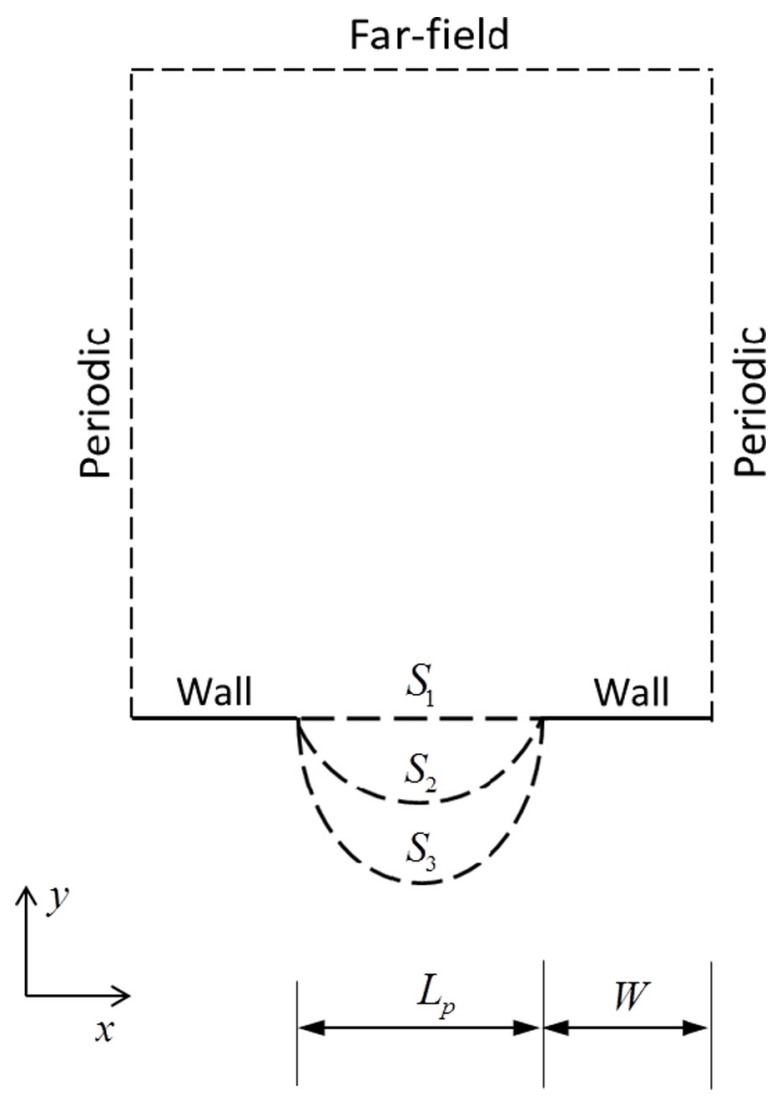

FIG. 1. Schematic of the computational geometry (not to scale).

rates have been found to be very sensitive to the choice of the evaporation coefficient specified. Therefore, we also investigate the dependence of the evaporation coefficient on the performance of nanoporous membranes in this work.

\section{PROBLEM FORMULATION AND SIMULATION DETAILS}

A schematic of the two-dimensional DSMC computational domain employed for carrying out the nanoporous evaporation simulations in this work is shown in Fig. 1. We consider a unit nanoporous membrane configuration, periodic in the $x$ direction, that is representative of the entire system of pores. The nanopore porosity is defined as $\phi=L_{p} /\left(2 W+L_{p}\right)$, where $L_{p}$ is the width of the pore and $W$ represents the width of the pore walls on either side of the meniscus. A porosity of unity represents the case with no walls, i.e., $W=0$. Variation in $\phi$ is achieved by fixing $L_{p}$, the characteristic length scale in our problem, and changing $W$. As illustrated in the figure, three meniscus shapes are considered: $S_{1}$ corresponds to a flat meniscus $(R=\infty), S_{2}$ corresponds to a curved meniscus $\left(R=0.4 L_{p}\right)$, and $S_{3}$ corresponds to a semicircle meniscus case $\left(R=0.5 L_{p}\right)$. The meniscus represents the liquid-vapor interface where evaporation takes place. The curved meniscus cases have a larger interfacial surface compared to the flat meniscus case, which we expect to influence the dynamics.

At the interface, a constant pressure, $P_{s}$, and temperature, $T_{s}$, are imposed. This is a reasonable assumption when the Biot number $\operatorname{Bi}\left(=L_{p} h_{\mathrm{e}} / 2 k_{1}\right) \ll 1$, where $h_{\mathrm{e}}$ is the evaporation heat transfer coefficient and $k_{1}$ is the liquid thermal conductivity. The Knudsen number is defined as $\mathrm{Kn}=\lambda / L_{p}$, 
where $\lambda$ is the mean free path based on the interface operating conditions. The variation in $\mathrm{Kn}$ is achieved by changing the operating pressure at the interface. The particle wall interactions are considered to be fully diffuse at a constant wall temperature, $T_{s}$.

At the interface (meniscus) boundary, a half-range Maxwellian is employed to specify the distribution function of evaporating molecules [48]:

$$
f_{e}(\boldsymbol{c})=\sigma \frac{n_{s}}{\left(2 \pi R T_{s}\right)^{3 / 2}} \exp \left(-\frac{\boldsymbol{c}^{2}}{2 \mathcal{R} T_{s}}\right), \quad \boldsymbol{c} \cdot \boldsymbol{n}>0 .
$$

Here $n_{s}$ is the saturated vapor number density and temperature at the interface; $\mathcal{R}$ is the specific gas constant and $\sigma$ the evaporation coefficient. The normal unit vector of the interface, pointing into the vapor, is denoted by $\boldsymbol{n}$. The Maxwell gas-surface scattering kernel, $J\left(\boldsymbol{c}^{\prime}, \boldsymbol{c}\right)$, is used to describe the molecular reemission from the interface, i.e., vapor molecules with initial velocity $\boldsymbol{c}$ is instantaneously changed to $\boldsymbol{c}^{\prime}$ upon interacting with the condensed phase at the interface:

$$
J\left(\boldsymbol{c}^{\prime}, \boldsymbol{c}\right)=(1-\sigma)\left[\alpha\left(\boldsymbol{c}^{\prime} \cdot \boldsymbol{n}\right) \frac{1}{2 \pi\left(\mathcal{R} T_{s}\right)^{2}} \exp \left(-\frac{\boldsymbol{c}^{\prime 2}}{2 \mathcal{R} T_{s}}\right)+(1-\alpha) \delta\left[\boldsymbol{c}^{\prime}-\boldsymbol{c}+2(\boldsymbol{c} \cdot \boldsymbol{n}) \boldsymbol{n}\right]\right] .
$$

The evaporation coefficient, $\sigma$, specifies the fraction of vapor molecules interacting with the interface and absorbed. Hence, $(1-\sigma)$ represents the total fraction of impinging molecules that are instantaneously reemitted, $\alpha$ is the probability of diffuse reemission, and $(1-\alpha)$ the probability of specular reflection. For all cases considered in this work, fully diffuse molecular reemissions are considered at the interface (i.e., $\alpha=1$ ).

The boundary conditions at the far-field (free-stream) boundary needs to be considered carefully, as the relations between the various free-stream parameters like density, velocity, and temperature are not known a priori. The equilibrium state attained by the vapor at the far field [48] can be described by the drifted Maxwellian distribution, $f_{\infty}(\boldsymbol{c})$ :

$$
f_{\infty}(\boldsymbol{c})=\frac{n_{\infty}}{\left(2 \pi R T_{\infty}\right)^{3 / 2}} \exp \left(-\frac{\left(\boldsymbol{c}-U_{\infty} \hat{\boldsymbol{y}}\right)^{2}}{2 \mathcal{R} T_{\infty}}\right) .
$$

Here, $n_{\infty}, T_{\infty}$, and $U_{\infty}$ are the number density, temperature and velocity, respectively, at free-stream conditions and $\hat{y}$ is the unit vector in the $y$ direction. For the evaporation problem, only one parameter can be freely specified; the other parameters need to be extracted as outcomes of this single driving parameter [24]. In this work, we fix the free-stream velocity as the single parameter that is imposed at the far-field boundary. For each case, we have ensured that the domain height $H$ is large enough for the vapor to reach free-stream equilibrium conditions. The free-stream velocity, $U_{\infty}$, has been imposed at the far field by forcing the velocity distribution function at the far-field boundary to be symmetric with respect to $U_{\infty}$ [37]. This is done by resetting the velocity of a particle crossing the far-field boundary as

$$
c^{\prime}=2 U_{\infty} \hat{\boldsymbol{y}}-\boldsymbol{c}
$$

such that

$$
F_{\infty}\left(\boldsymbol{c}^{\prime}\right)=F_{\infty}\left(2 U_{\infty} \hat{\boldsymbol{y}}-\boldsymbol{c}\right)=F_{\infty}(\boldsymbol{c}),
$$

where $\boldsymbol{c}$ and $\boldsymbol{c}^{\prime}$ are the particle velocity before and after interacting with the free-stream boundary. When a particle with velocity $c_{y}$ interacts with the far-field boundary, it is reassigned a velocity $c_{y}^{\prime}$ with a probability $\left|c^{\prime}{ }_{y}\right| / c_{y}$, if $c_{y}^{\prime}<0$.

All simulations in this work have been carried out using SPARTA [49], which is a recently developed, highly scalable parallel open-source DSMC code [50,51]. To enable the nanoporous evaporation simulations, we have implemented the interface evaporation [Eqs. (1) and (2)] and 


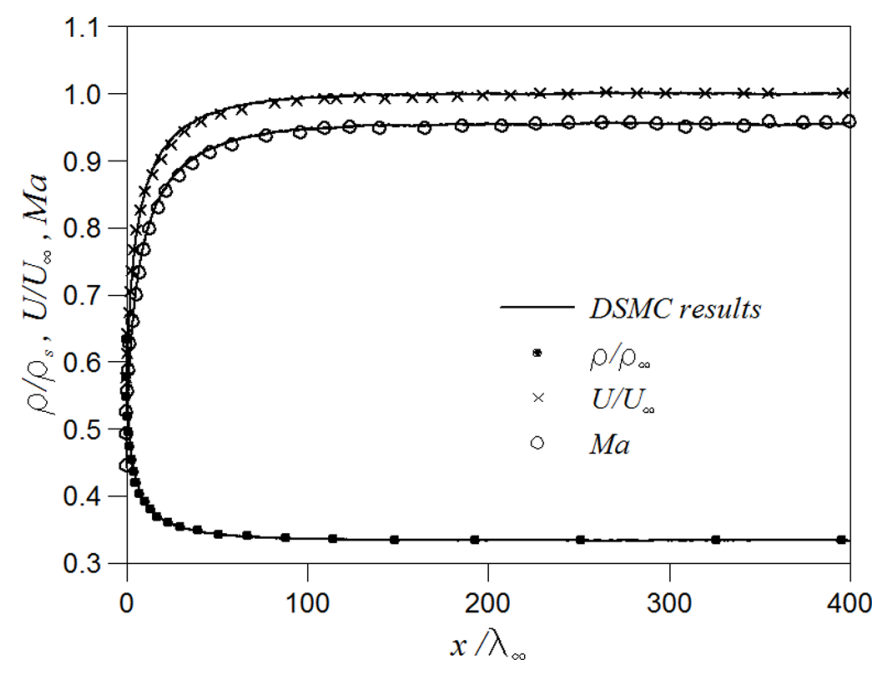

FIG. 2. Profiles of normalized density, velocity, Mach number, and mass flux at $\mathrm{Ma}_{\infty}=0.956$. Symbols denote data from literature [37] and lines denote the current DSMC results.

far-field free-stream velocity [Eqs. (3) and (4)] boundary conditions in SPARTA. The gas was assumed to be argon and the variable hard sphere (VHS) model was employed. Additionally, we have followed the well-known guidelines with respect to the cell size, time step, and particle numbers that are needed for accurate DSMC calculations [32,52]. The time step for the DSMC simulations is taken to be about five times smaller than $\Delta x_{\min } /\left(V_{m p}+U_{\infty}\right)$, where $\Delta x_{\min }$ is the smallest cell dimension and $V_{m p}$ is the most probable molecular velocity, given by $V_{m p}=\sqrt{2 \mathcal{R} T_{s}}$. To minimize statistical noise, an average of at least one hundred particles per cell has been considered and the sampling phase has been carried out over a period of at least a million time steps.

\section{RESULTS AND DISCUSSION}

In this section, validation of the code for the evaporation problem will be considered first (Sec. III A). This will be followed by nanoporous membrane simulations considering different porosities, interface Knudsen numbers, and free-stream Mach numbers (Sec. III B). In particular, the ideal case of $\phi=1$ will be considered, followed by nonunity porosities. The evaporation rates, the density ratios, and the temperature jumps will be investigated as a function of different operating conditions. To gain an understanding of the physical mechanisms at work, the flow physics will be further explored in Sec. III C by investigating the flow fields and evaporation rates of selected cases in detail. Section III D will consider the impact of the evaporation coefficient on the evaporation rates, while Sec. III E deals with the efficacy of the receding meniscus for selected cases.

\section{A. Validation}

Validation of the code has been performed against benchmark results from the literature for two selected cases. Both cases are particularly relevant to the current study: they consider evaporation from a planar surface in the one-dimensional limit. The first case considers evaporation from a planar surface at a selected free-stream Mach number, $\mathrm{Ma}_{\infty}=0.956$, which is defined as $U_{\infty} / \sqrt{\kappa \mathcal{R} T_{\infty}}$, where $\kappa$ is the specific heat ratio. The comparison of the computed normalized profiles for density, velocity, and Mach number against corresponding results in the literature (Frezzotti [37]) is shown in Fig. 2. The figure shows the variation of the flow parameters across the Knudsen layer and into the far-field region. One can clearly see the density drop and velocity rise (corresponding to a flow 


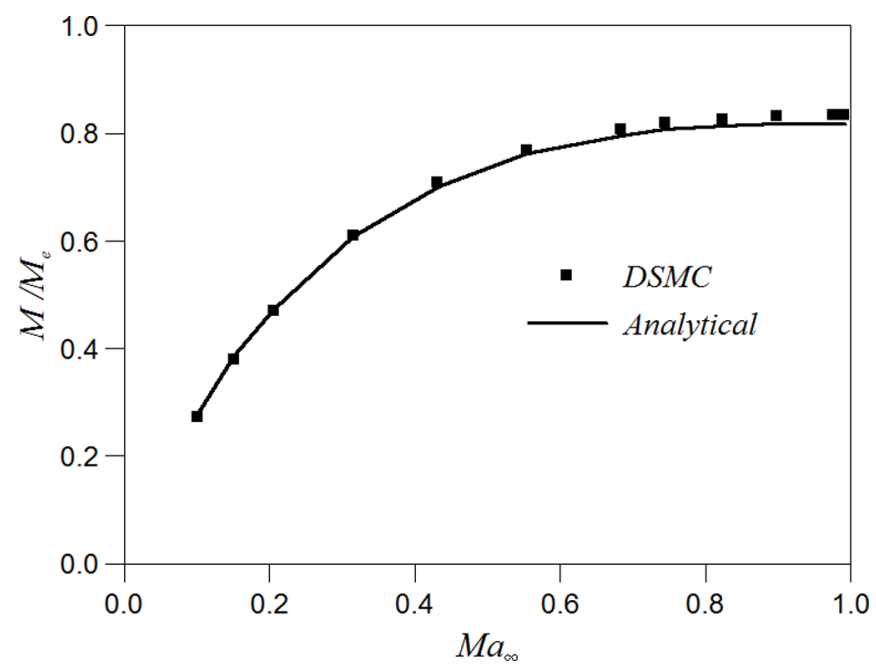

FIG. 3. Validation of the evaporative mass flux against analytical results from Labuntsov and Kryukov [28].

expansion) in the Knudsen layer before the flow attains the imposed equilibrium free-stream velocity conditions further away from the interface. Excellent agreement with results from [37] can be noted for all computed quantities.

Validation has also been done for the evaporative mass flux from a planar surface against analytical solutions given by Labuntsov and Kryukov [28]. The one-dimensional analytical mass flux, $M_{1 \mathrm{D}}$ is given by

$$
M_{1 \mathrm{D}}=0.6 C_{s}\left(\frac{\rho_{\infty}}{\rho_{0}}\right)^{1 / 2}\left(\rho_{0}-\rho_{\infty}\right)
$$

where the interface density is expressed as

$$
\rho_{0}=\left(1-2 \sqrt{\pi} \frac{M_{1 D}}{\rho_{s} C_{s}} \frac{1-\sigma}{\sigma}\right) \rho_{s}
$$

Here, $\sigma$ is the evaporation coefficient and $C_{s}=\sqrt{2 \mathcal{R} T_{s}}$. The terms $\rho_{s}$ and $\rho_{\infty}$ are the saturation and free-stream densities, respectively. The validation is shown in Fig. 3 for $\sigma=1$, where the mass flux values have been normalized with respect to the analytical expression for total emitted (evaporative) mass flux from a given surface, $M_{e}$, given by

$$
M_{e}=\rho_{s} \sqrt{\frac{\mathcal{R} T_{s}}{2 \pi}} .
$$

The mass flux is assessed near the far field and has been calculated as $M=\rho_{\infty} U_{\infty}$. Comparison of the normalized mass flux values has been made for a range of free-stream Mach numbers and the results are shown in Fig. 3. It can be noted that our simulations are in good agreement with the analytical results, with minor deviations only near the sonic flow limit. The analytical moment solution predicts a critical mass flux of 0.818 , whereas the DSMC solutions predict a critical mass flux of 0.835 . The discrepancy is due to the slight inaccuracy of the moment solution near the sonic flow limit, which is an issue previously reported in the literature [24]. No such issues occur for the more accurate mass flux solutions obtained using the DSMC method [33,34] and numerical solutions of the BGK model equation by Sone et al. [30], so that, as expected, our critical mass flux value of 0.835 is in excellent agreement with previous DSMC and BGK model solutions [30,33,34]. 


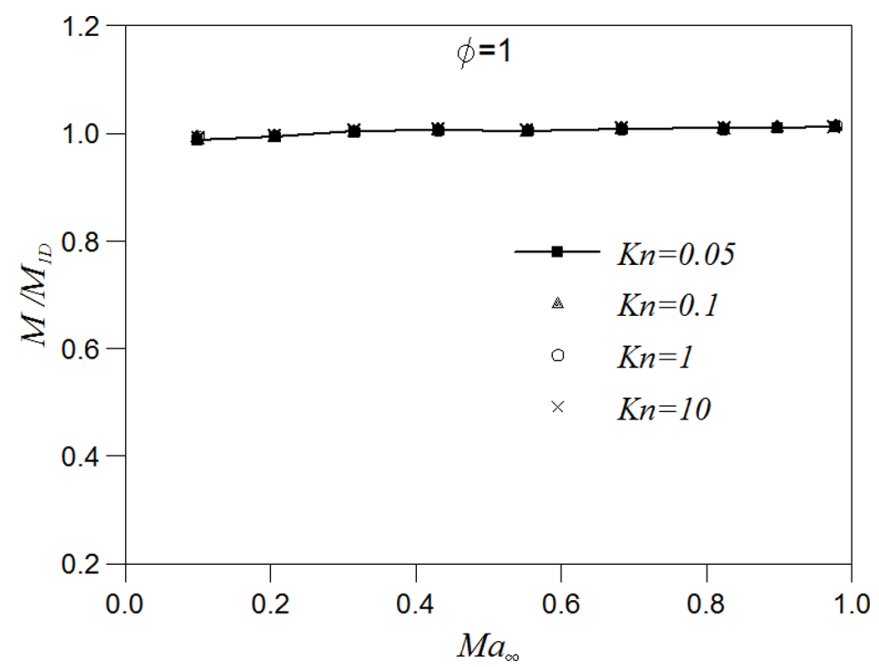

FIG. 4. Computed mass flux versus the free-stream Mach number for the flat meniscus case, $S_{1}$ at $\phi=1$.

\section{B. Nanoporous membrane simulations}

Consider, initially, evaporation from different meniscus cases for porosity $\phi=1$. Porosity of unity represents the ideal case, as it has the maximum available evaporative area, and thus favors large heat flux dissipation rates. However, note that in reality, the maximum porosity that can be attained is subject to membrane manufacturing, mechanical integrity, and conduction heat-transport considerations. Nevertheless, the case $\phi=1$ is of particular interest to study as the limiting case where supporting walls are extremely thin $(W \rightarrow 0)$. The evaporation rate from the flat meniscus, $S_{1}$, is investigated first for a wide range of free-stream Mach numbers and Knudsen numbers. Figure 4 shows the variation in the computed mass flux with Mach number for this case. Four Knudsen numbers are considered. The mass flux values, assessed at the far-field boundary, have been normalized with respect to the evaporative mass flux from a planar surface, $M_{1 D}$, given by Eq. (6), which should be exact in this case. For all cases of Kn considered, the mass flux increases with Mach number before reaching a critical value near sonic free-stream conditions (i.e., $M a_{\infty}=1$ ). The flat meniscus case corresponds to the classic case of evaporation from a planar surface, for which the flow features are essentially one-dimensional. Therefore, as expected, the predicted normalized evaporation rates for all flat meniscus cases are unity and are independent of the Knudsen number considered.

The evaporation rates for the curved meniscus cases, $S_{2}$ and $S_{3}$, relative to the flat meniscus case are examined next for $\phi=1$. Figure 5 shows the variation in the computed mass flux for the curved meniscus cases for different Mach numbers and Knudsen numbers. The mass flux values here have been normalized with respect to the evaporative mass flux from a planar surface, $M_{1 D}$, given by Eq. (6). It can be noted that the predicted evaporation rates are clearly dependent on the curvature shape and also on the Knudsen number. The curved menisci have a higher surface area in comparison to the flat case; however, a larger surface area also implies a greater probability for molecular losses through the interface, so that the result of these two competing effects is nontrivial. The net evaporative mass flux is determined by the balance between molecular emission and condensation-reemission phenomenon at the liquid-vapor interface, which in turn is dependent on the operating conditions considered.

Low free-stream Mach numbers are characterized by low evaporation rates, as expected. More interestingly, at very low evaporation rates, all meniscus shapes behave identically, as noted from their evaporation rates. The variation of the normalized mass flux values with change in the freestream Mach number shows interesting trends. At low values of $\mathrm{Kn}$ (i.e., $\mathrm{Kn}=0.05$ ), the relative mass flux increases with flow speed for both curved menisci. A critical mass flux is attained near 


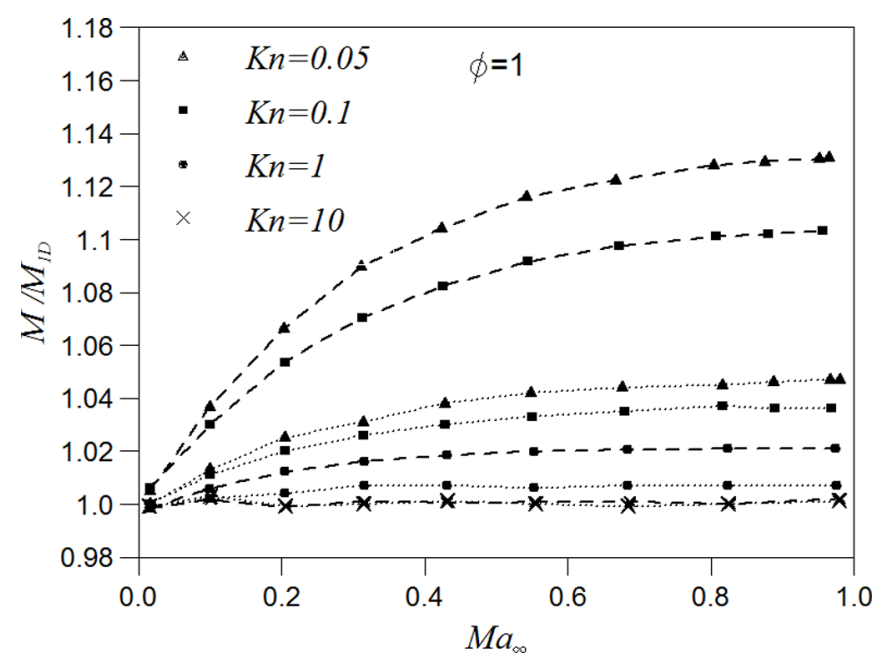

FIG. 5. Normalized mass flux versus the free-stream Mach number for the curved meniscus cases at $\phi=1$. Dashed lines represent the semicircle meniscus, $S_{3}$; dotted lines represent the curved meniscus, $S_{2}$.

sonic flow conditions, similar to that noted for evaporation from a planar surface. The maximum increase in mass flux (relative to the flat meniscus case) is observed for the semicircle meniscus, as it has a relatively larger surface area compared to the curved meniscus case, $S_{2}$.

As $\mathrm{Kn}$ increases, the variations in the normalized mass flux with increase in free-stream flow velocity become relatively minimal. As the flow enters the free molecular regime (i.e., $\mathrm{Kn}=10$ ), the predicted relative mass flux shows no variation with $\mathrm{Ma}_{\infty}$; i.e., the computed mass fluxes for the curved meniscus cases are identical to those predicted for the flat meniscus case. This is because at relatively high Knudsen numbers there is a greater probability of molecular condensation at the interface due to the fact that the collision frequency decreases.

The variations in the corresponding density $\left(\rho_{\infty} / \rho_{s}\right)$ and temperature $\left(T_{\infty} / T_{s}\right)$ drops for the different meniscus cases considered are illustrated in Fig. 6. Three selected Knudsen numbers are considered, i.e., $\mathrm{Kn}=0.05,1$, and 10 . It is worth noting that only a single parameter, i.e., the free-stream velocity, is imposed, and therefore for a constant $U_{\infty}$ the Mach number varies in accordance with the computed free-stream temperature. It can be noted that, for all degrees of flow rarefaction considered, the drop in temperature ratio (temperature jump) varies almost linearly as $\mathrm{Ma}_{\infty}$ increases. The density, on the other hand, drops more rapidly and varies in a nonlinear fashion with the Mach number. In general, the highest density ratio and temperature jumps have been obtained for the semicircle meniscus, $S_{3}$, followed by the curved meniscus, $S_{2}$. However, these differences are discernible only near the slip and continuum regimes (i.e., at relatively low values of $\mathrm{Kn}$ ). In the upper transition and free molecular regimes (i.e., $\mathrm{Kn} \geqslant 1$ ), the deviations are minimal. This is consistent with the trend in the variation of mass flux (noted in Fig. 5), where the semicircle meniscus case attains the maximum mass flux, particularly at low values of Kn.

We now examine the evaporation rates when porosity is less than unity $(\phi<1)$ for a range of Knudsen numbers and free-stream Mach numbers. Figure 7 shows the variation in computed mass flux as a function of Mach number for three selected Knudsen numbers (i.e. $\mathrm{Kn}=0.05,1$, and 10). Two values of porosity are considered, i.e., $\phi=0.75$ and 0.5 . The mass flux values here have again been benchmarked against the ideal case of evaporative mass flux from a planar surface, $M_{1 \mathrm{D}}$, given in Eq. (6), for a consistent comparison. It can be noted that, for very low evaporation rates (corresponding to low free-stream Mach numbers), changes to porosity do not significantly affect the mass flux values. However, as $\mathrm{Ma}_{\infty}$ increases, reductions in the value of porosity can lead to substantial reductions in mass flux. This can be attributed mainly to the relative decrease 


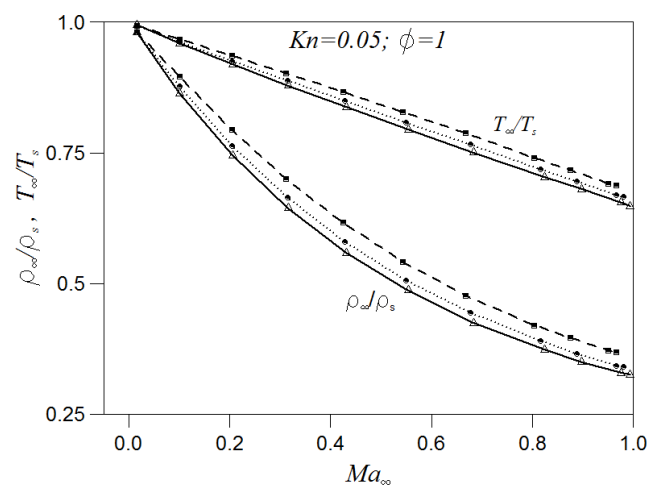

(a)

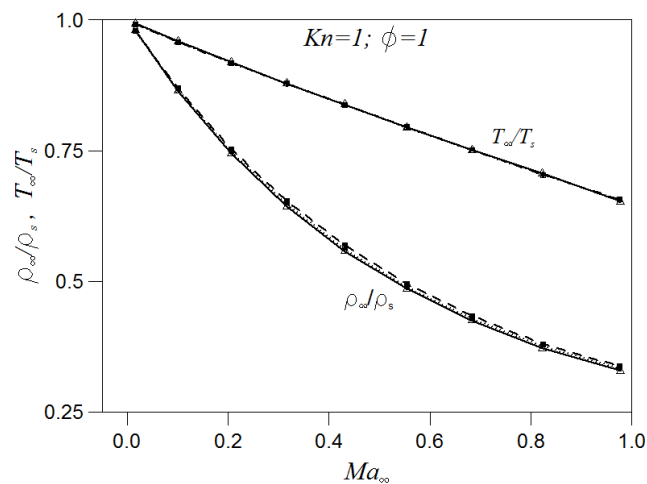

(b)

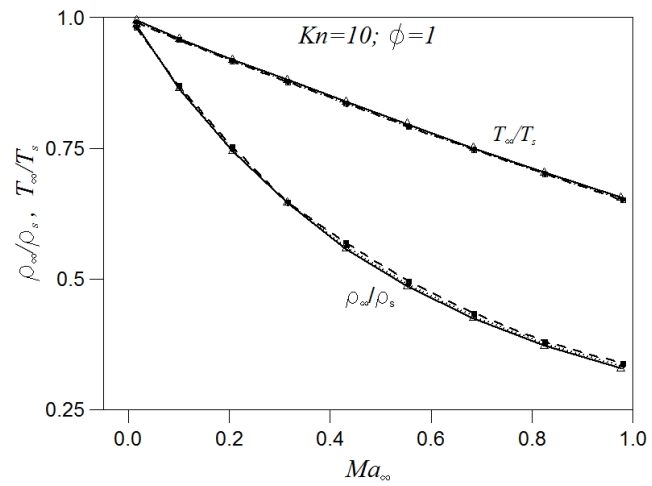

(c)

FIG. 6. Computed density ratios and temperature jumps as a function of the free-stream Mach number at $\phi=1$. Dashed lines represent semicircle meniscus, $S_{3}$; dotted lines represent the curved meniscus, $S_{2}$; and solid lines represent the flat meniscus, $S_{1}$.

in the available evaporative surface as porosity decreases. However, this is not the only factor, as the proportion of reduction in mass flux depends significantly on the free-stream conditions under consideration. At low $\mathrm{Ma}_{\infty}$, the reduction in mass flux is significantly lower when compared to that at a higher free-stream Mach number. This is mainly due to the increased role of gas expansion near the interface as $\mathrm{Ma}_{\infty}$ increases. The role of gas expansion with changing operating conditions will be dealt with in more detail in Sec. III C.

It can be also noted from Fig. 7 that the variation in the predicted evaporation rates for the different menisci considered depends on the Knudsen number. In the upper transition and free molecular regimes (i.e., $\mathrm{Kn} \geqslant 1$ ), the differences in the predicted evaporation rates between the different meniscus shapes considered are minimal for all values of free-stream velocities considered. However, at low degrees of flow rarefaction, significant differences in mass flux can be noted between the meniscus cases, particularly so at the higher free-stream velocities considered. For instance, at $\mathrm{Kn}=0.05$, the semicircle meniscus, $S_{3}$, attains the largest relative increase in evaporation rates followed by the curved meniscus case, $S_{2}$. It can also be noted that a critical mass flux is attained near sonic free-stream flow conditions $\left(\right.$ as $\left.\mathrm{Ma}_{\infty} \rightarrow 1\right)$ for both cases of porosity considered. In particular, the critical mass flux value $\left(M / M_{1 D}\right)$ attained for the flat meniscus case can be noted to be very similar for all Knudsen numbers; for $\phi=0.75$ the corresponding critical mass flux is about 0.784 and for $\phi=0.5$ it is about 0.548 .

The drops in the corresponding density ratios and temperature jumps with increase in free-stream Mach number for $\phi=0.75$ and 0.5 are illustrated in Fig. 8. It can be noted that the trends in 


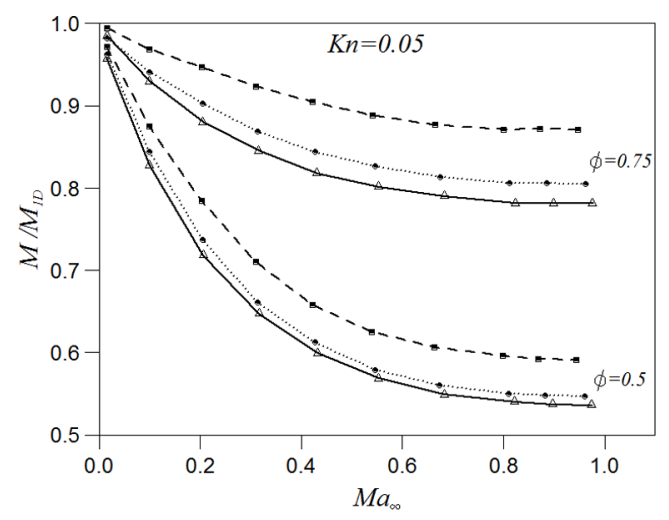

(a)

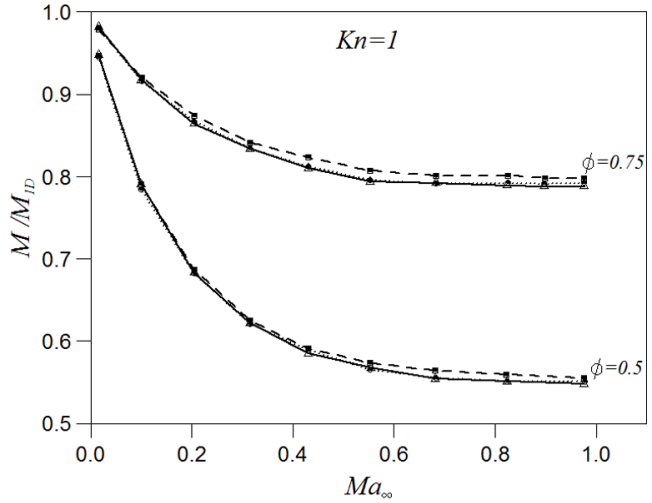

(b)

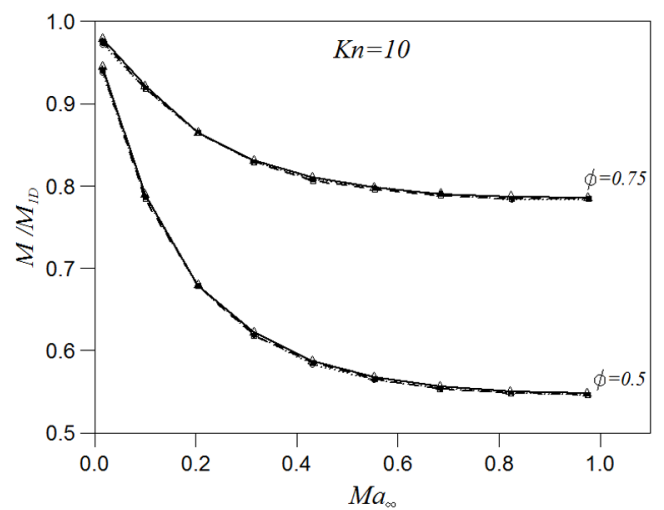

(c)

FIG. 7. Normalized mass flux as a function of the free-stream Mach number for three selected Knudsen numbers when $\phi<1$. Dashed lines represent the semicircle meniscus, $S_{3}$; dotted lines represent the curved meniscus, $S_{2}$; and solid lines represent the flat meniscus, $S_{1}$.

the variations are similar to that observed for $\phi=1$, and also are consistent with the mass flux predictions. The variations between different meniscus cases are again minimal for high Knudsen numbers. Interestingly, unlike density ratios, the temperature jumps are not affected notably with reduction in porosity, especially at higher degrees of flow rarefaction.

Before concluding this section, we also provide empirical expressions for the evaporative mass flux, density ratio, and pressure ratio for selected conditions by curve fitting the available data. In particular, the free molecular regime $(\mathrm{Kn}=10)$ has been chosen for this purpose, as optimized nanoporous configurations typically work best in the high Knudsen number regime. Density and pressure ratios are first expressed as a function of the free-stream Mach number [Eqs. (9)-(14)]. Mass flux values, $M / M_{1 D}$, are then expressed as a function of $\rho_{\infty} / \rho_{s}$ and $P_{\infty} / P_{s}$ [Eqs. (15)-(18)], as density and pressure ratios are usually the most relevant input parameters of interest. The corresponding empirical expressions for $\mathrm{Kn}=10$ at different porosities for the flat meniscus case are given below; note that, for a porosity of $1, M / M_{1 D}$ approaches unity and therefore the corresponding expression has not been provided:

$$
\begin{aligned}
& \left.\frac{\rho_{\infty}}{\rho_{s}}\right|_{\phi=1}=0.115 \mathrm{Ma}_{\infty}^{4}-0.602 \mathrm{Ma}_{\infty}^{3}+1.286 \mathrm{Ma}_{\infty}^{2}-1.476 \mathrm{Ma}_{\infty}+1.0, \\
& \left.\frac{\rho_{\infty}}{\rho_{s}}\right|_{\phi=0.75}=1.084 \mathrm{Ma}_{\infty}^{4}-3.156 \mathrm{Ma}_{\infty}^{3}+3.7 \mathrm{Ma}_{\infty}^{2}-2.373 \mathrm{Ma}_{\infty}+1.0,
\end{aligned}
$$




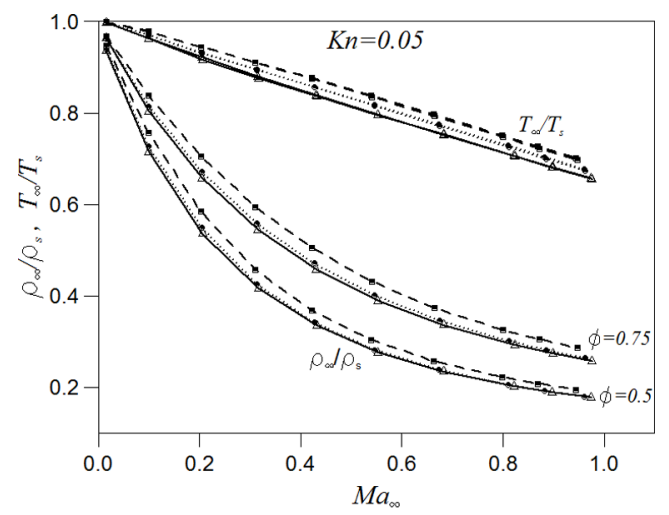

(a)

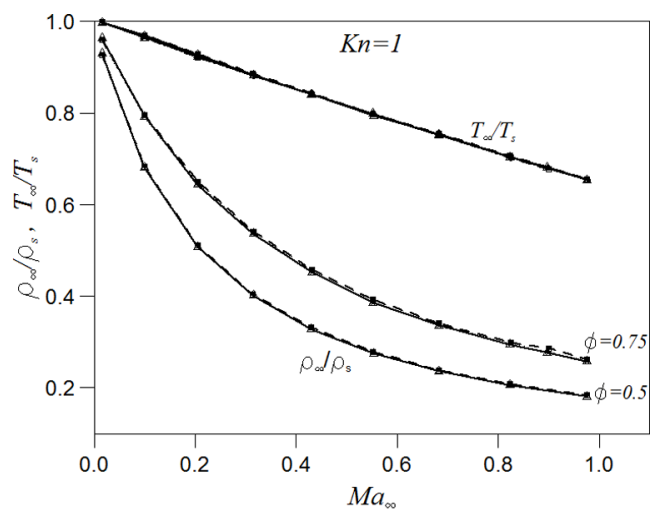

(b)

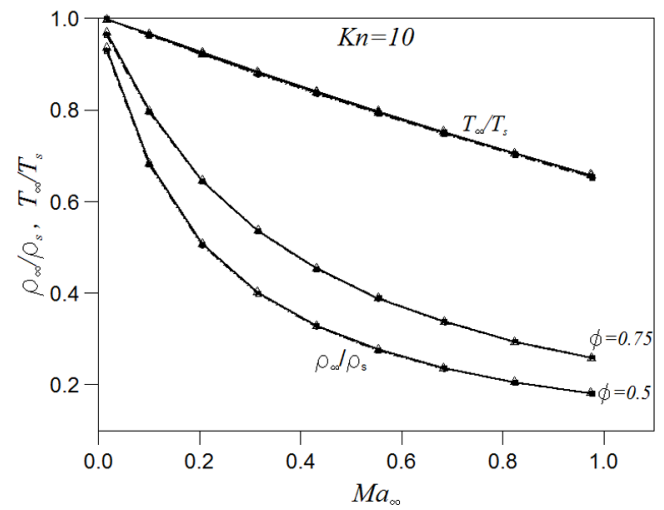

(c)

FIG. 8. Computed density ratios and temperature jumps as a function of the free-stream Mach number for three selected Knudsen numbers when $\phi<1$. Dashed lines represent the semicircle meniscus, $S_{3}$; dotted lines represent the curved meniscus, $S_{2}$; and solid lines represent the flat meniscus, $S_{1}$.

$$
\begin{gathered}
\left.\frac{\rho_{\infty}}{\rho_{s}}\right|_{\phi=0.5}=3.313 \mathrm{Ma}_{\infty}^{4}-8.450 \mathrm{Ma}_{\infty}^{3}+8.041 \mathrm{Ma}_{\infty}^{2}-3.71 \mathrm{Ma}_{\infty}+1.0, \\
\left.\frac{P_{\infty}}{P_{s}}\right|_{\phi=1}=0.361 \mathrm{Ma}_{\infty}^{4}-1.27 \mathrm{Ma}_{\infty}^{3}+2.013 \mathrm{Ma}_{\infty}^{2}-1.895 \mathrm{Ma}_{\infty}+1.0, \\
\left.\frac{P_{\infty}}{P_{s}}\right|_{\phi=0.75}=1.626 \mathrm{Ma}_{\infty}^{4}-4.477 \mathrm{Ma}_{\infty}^{3}+4.865 \mathrm{Ma}_{\infty}^{2}-2.846 \mathrm{Ma}_{\infty}+1.0 \\
\left.\frac{P_{\infty}}{P_{s}}\right|_{\phi=0.5}=3.883 \mathrm{Ma}_{\infty}^{4}-9.795 \mathrm{Ma}_{\infty}^{3}+9.161 \mathrm{Ma}_{\infty}^{2}-4.112 \mathrm{Ma}_{\infty}+1.0 \\
\left.\frac{M}{M_{1 D}}\right|_{\phi=0.75}=-0.391\left(\frac{\rho_{\infty}}{\rho_{s}}\right)^{4}+0.502\left(\frac{\rho_{\infty}}{\rho_{s}}\right)^{3}+0.222\left(\frac{\rho_{\infty}}{\rho_{s}}\right)^{2}-0.147\left(\frac{\rho_{\infty}}{\rho_{s}}\right)+0.8 \\
\left.\frac{M}{M_{1 D}}\right|_{\phi=0.5}=0.705\left(\frac{\rho_{\infty}}{\rho_{s}}\right)^{4}-2.19\left(\frac{\rho_{\infty}}{\rho_{s}}\right)^{3}+2.5\left(\frac{\rho_{\infty}}{\rho_{s}}\right)^{2}-0.619\left(\frac{\rho_{\infty}}{\rho_{s}}\right)+0.6
\end{gathered}
$$




$$
\begin{gathered}
\left.\frac{M}{M_{1 D}}\right|_{\phi=0.75}=-0.2\left(\frac{P_{\infty}}{P_{s}}\right)^{4}+0.087\left(\frac{P_{\infty}}{P_{s}}\right)^{3}+0.415\left(\frac{P_{\infty}}{P_{s}}\right)^{2}-0.1\left(\frac{P_{\infty}}{P_{s}}\right)+0.79, \\
\left.\frac{M}{M_{1 D}}\right|_{\phi=0.5}=0.516\left(\frac{P_{\infty}}{P_{s}}\right)^{4}-1.615\left(\frac{P_{\infty}}{P_{s}}\right)^{3}+1.796\left(\frac{P_{\infty}}{P_{s}}\right)^{2}-0.263\left(\frac{P_{\infty}}{P_{s}}\right)+0.56 .
\end{gathered}
$$

\section{Physical mechanisms at work}

To gain further insight into the physical mechanisms at work, we now study the flow fields and the evaporation rates for selected cases as a function of the Knudsen number. First, we show representative flow fields to demonstrate the Mach number, density ratio, and temperature jump variation next to the interface. The flow fields are for selected cases, i.e., the flat meniscus (Fig. 9) and semicircle meniscus (Fig. 10) for fixed porosity $\phi=0.5$ and free-stream velocity $U_{\infty}=30 \mathrm{~m} / \mathrm{s}$ but different degrees of flow rarefaction, $\mathrm{Kn}=0.05,0.1$, and 10 . The flow fields suggest a flow expansion phenomenon in the Knudsen layer next to the interface. The flow expansion specifically implies a gas density drop in the Knudsen layer before it attains the downstream equilibrium density conditions and a corresponding temperature drop and velocity rise in accordance with this density drop. The basic flow expansion features near the interface for all degrees of flow rarefaction can be noted to be largely similar. A density drop, velocity rise, and temperature drop corresponding to the gas expansion can be clearly noted near the interface for all cases. Further away from the interface, downstream equilibrium conditions are attained, and there are minimal variations of the flow properties in the spanwise $(x)$ direction. To illustrate the variation in density ratio, Mach number, and temperature drop along the extent of the physical domain (in the $y$ direction) more clearly, additional plots are shown in Fig. 11 for the semicircle meniscus case. It can be noted that, although the nature of flow expansion is qualitatively the same for all degrees of flow rarefaction, the downstream equilibrium conditions (particularly, the density ratios) for lower Knudsen numbers are attained at a relatively shorter distance from the interface when compared to those at higher Kn values. Therefore, the higher Kn cases need a relatively larger domain height $H$. Furthermore, it can be noted from Fig. 11 that higher Knudsen numbers are characterized by a larger density ratio, a smaller Mach number increase and a lower free-stream temperature, relative to those at lower Knudsen numbers for the same conditions considered.

The computed mass flux is examined next as a function of Knudsen number $(0.025<\mathrm{Kn}<10)$ and porosity $(0.5<\phi<1)$. Whereas the previous section considered evaporation rates as a function of free-stream Mach number at fixed values of Knudsen number, here we fix the free-stream velocity and vary the Knudsen number to gain additional insights. Three selected free-stream velocities are considered here, i.e., $U_{\infty}=30,60$, and $180 \mathrm{~m} / \mathrm{s}$, and the corresponding results are shown in Fig. 12. It can be clearly noted again that a reduction in porosity can lead to substantial losses and reduction in the evaporation rates. Moreover, it can be observed that the proportion by which the evaporation rates reduce with decreasing porosity increases significantly as the free-stream velocity increases. For instance, in the free molecular regime, the relative reductions in the evaporation rate when $\phi=0.5$ are about $20 \%, 30 \%$, and $44 \%$ for $U_{\infty}=30,60$, and $180 \mathrm{~m} / \mathrm{s}$, respectively. The reduction in the evaporation rates as porosity decreases and free-stream Mach number increases can be attributed to the fact that there is a relatively larger flow expansion and, correspondingly, a larger pressure drop at higher free-stream velocities. To illustrate the flow expansion near the interface with increase in $\mathrm{Ma}_{\infty}$ more explicitly, the density ratio $\rho_{\infty} / \rho_{s}$ is plotted along the extent of the computational domain for a selected Knudsen number, $\mathrm{Kn}=10$, at different free-stream velocities $U_{\infty}=30,60$, and $180 \mathrm{~m} / \mathrm{s}$ in Fig. 13 for the flat meniscus case. The sudden drop in density near the interface, before it reaches the density value corresponding to the downstream equilibrium conditions, can be noted. Clearly, higher $\mathrm{Ma}_{\infty}$ leads to larger density drops. The temperature drop and velocity rise corresponding to the density drop before it reaches the downstream equilibrium conditions can also be clearly noted from Fig. 13. Additionally, as $\mathrm{Ma}_{\infty}$ increases, the downstream equilibrium conditions are attained farther away from the interface. We also analyze the dependence of porosity 
(a)

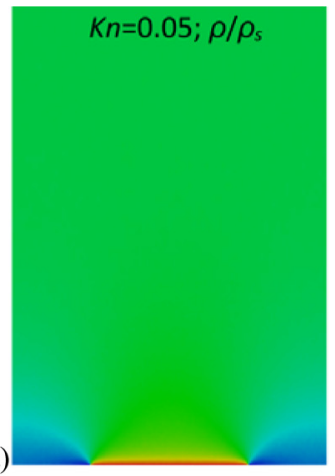

(b)

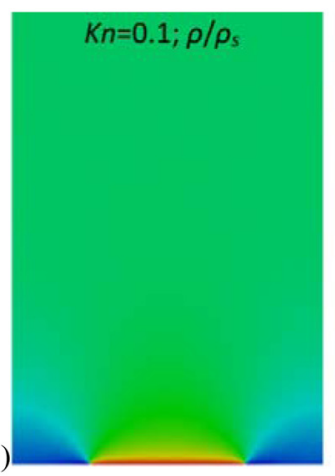

(c)

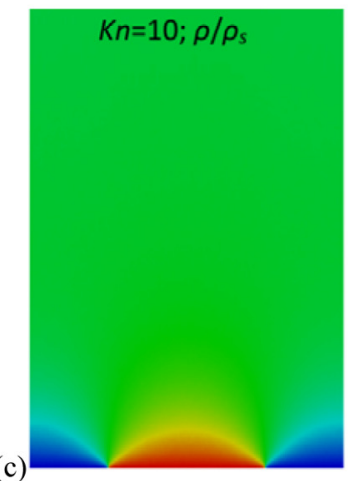

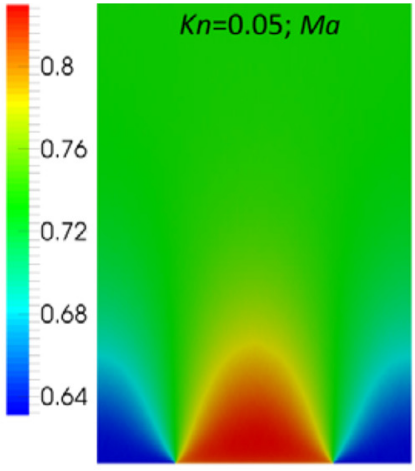
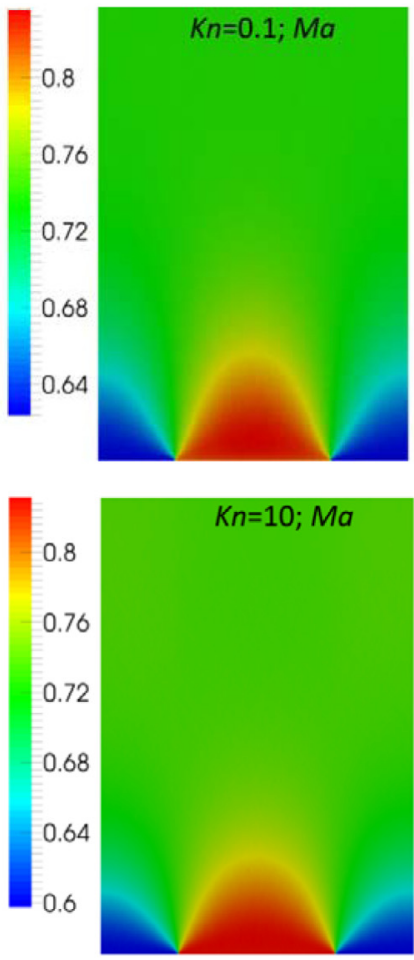

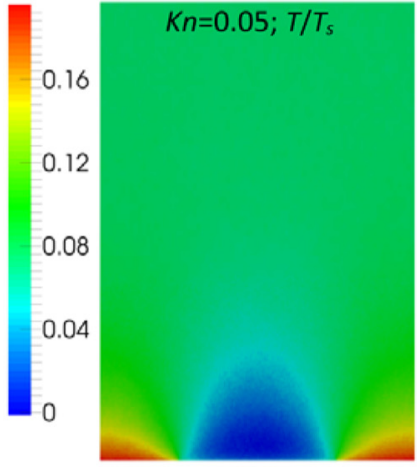

0.98

0.96

0.94

0.92

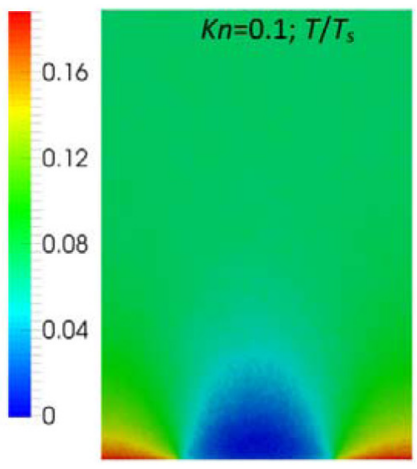

0.98

0.96

0.94

0.92

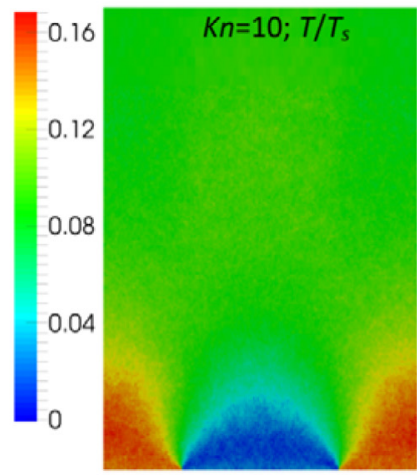

0.96

0.95

0.94

FIG. 9. Contour plots showing variation of $\rho / \rho_{s}$, Ma, and $T / T_{s}$ (respectively from left to right) for the flat meniscus, $S_{1}$. The flow fields shown are for a fixed porosity $\phi=0.5$ and free-stream velocity $U_{\infty}=30 \mathrm{~m} / \mathrm{s}$, but different degrees of flow rarefaction: (a) $\mathrm{Kn}=0.05$, (b) $\mathrm{Kn}=0.1$, and (c) $\mathrm{Kn}=10$. Only a selected portion of the computational domain is shown for each case.

on the flow expansion behavior near the interface by plotting the relevant flow parameters for a selected fixed Knudsen number, $\mathrm{Kn}=10$, for the flat meniscus case. The variations in the flow quantities for the different porosities considered are shown in Fig. 14. It can be noted that a reduction in porosity is characterized by substantial density drops. The corresponding changes to the velocity rise and temperature drop are minimal.

A further observation from the evaporation rates versus Kn plot in Fig. 12 is that in the upper transition and free molecular regimes $(\mathrm{Kn} \geqslant 1)$, the differences in the predicted evaporation rates between the different meniscus shapes considered are minimal, for all values of $U_{\infty}$ and 

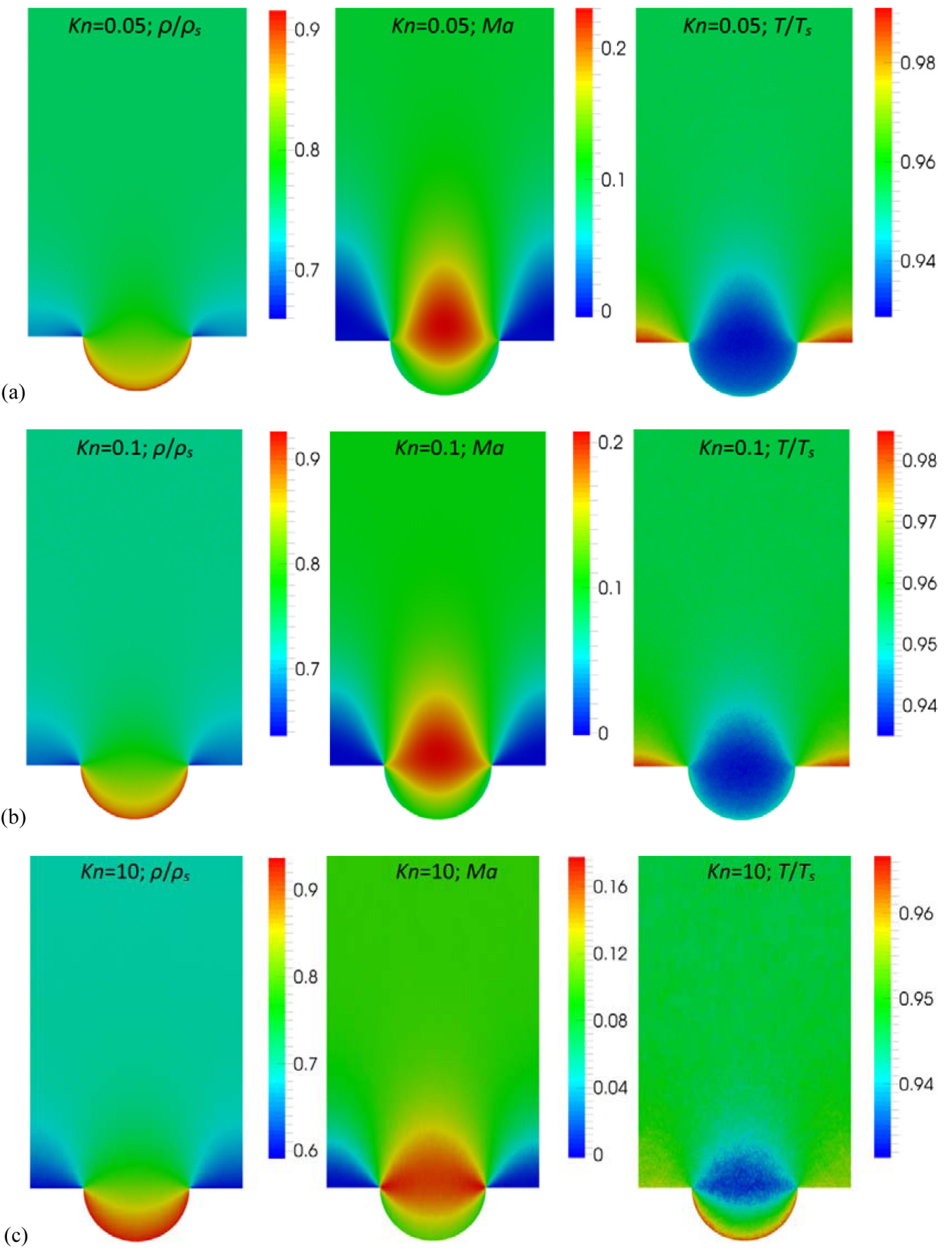

FIG. 10. Contour plots showing variation of $\rho / \rho_{s}$, Ma, and $T / T_{s}$ (respectively from left to right) for the semicircle meniscus, $S_{3}$. The flow fields shown are for a fixed porosity $\phi=0.5$ and free-stream velocity $U_{\infty}=30 \mathrm{~m} / \mathrm{s}$, but different degrees of flow rarefaction: (a) $\mathrm{Kn}=0.05$, (b) $\mathrm{Kn}=0.1$, and (c) $\mathrm{Kn}=10$. Only a selected portion of the computational domain is shown for each case. 


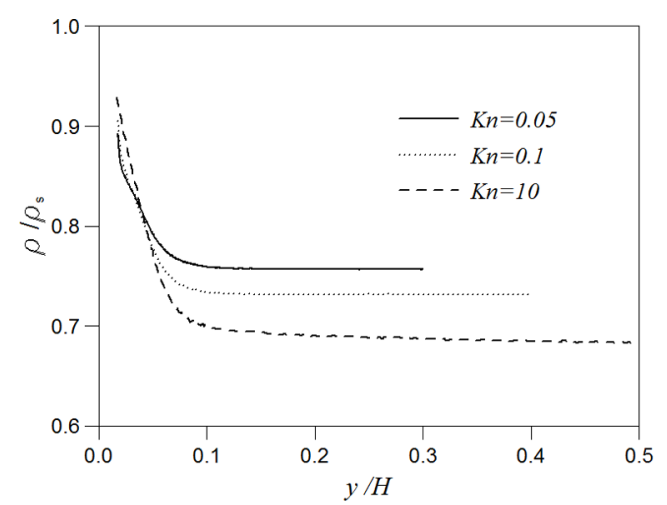

(a)

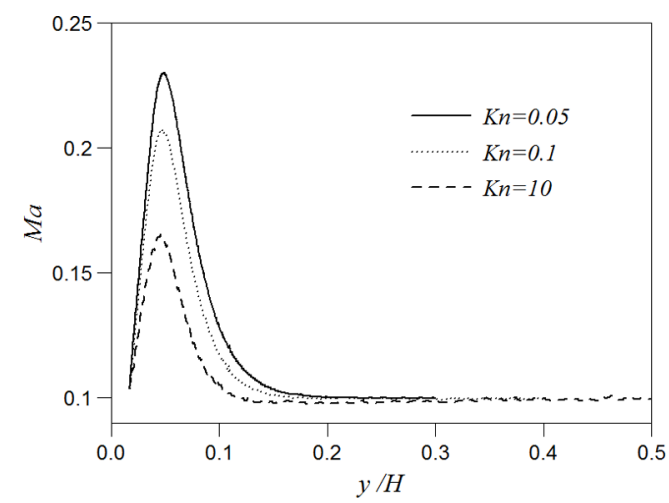

(b)

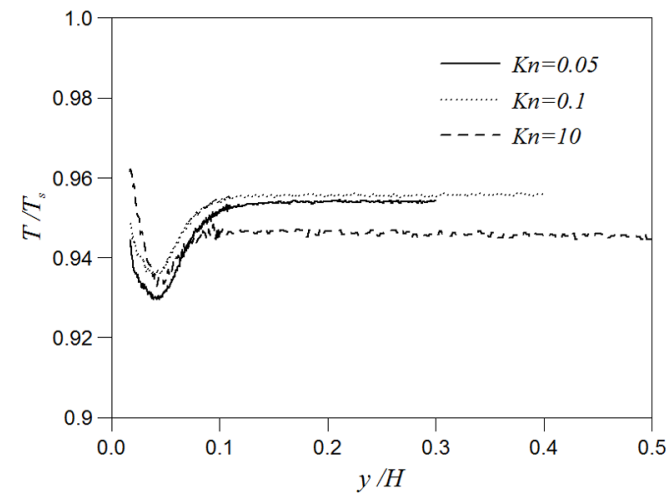

(c)

FIG. 11. Variation in (a) $\rho / \rho_{s}$, (b) Ma, and (c) $T / T_{s}$ plotted along the extent of the computational domain at $U_{\infty}=30 \mathrm{~m} / \mathrm{s}$ and different degrees of flow rarefaction.

$\phi$ considered. However, for lower degrees of flow rarefaction $(\mathrm{Kn} \leqslant 0.1)$, significant differences in mass flux can be noted between the meniscus cases, particularly so at higher free-stream velocities considered. For instance, the semicircle meniscus, $S_{3}$, attains the largest relative increase in evaporation rates, notably in the early slip/continuum regime. At $\mathrm{Kn}=0.025$ and $\phi=1$, the relative increases in computed mass flux for the semicircle meniscus (with respect to the flat meniscus case) are about $5.6 \%, 7.4 \%$, and $15 \%$, respectively, for $U_{\infty}=30,60$, and $180 \mathrm{~m} / \mathrm{s}$. The increase in relative mass flux as $U_{\infty}$ increases (for the same $\mathrm{Kn}$ ) can be attributed to the fact that, at higher flow velocities near the interface, there is relatively a higher probability for most molecules to travel unidirectionally (i.e., in the flow direction) and for correspondingly fewer molecules to travel in the opposite direction (i.e., towards the interface), resulting in a relatively smaller chance of condensation at the interface.

Additionally, Fig. 12 suggests that the proportion by which the mass flux reduces as porosity decreases is relatively lower near the early slip regime in comparison with the free molecular regime, particularly so at the low values of free-stream velocity considered. For instance, for the semicircle meniscus case considered at $U_{\infty}=30 \mathrm{~m} / \mathrm{s}$ and $\phi=0.5$ the reduction in mass flux at $\mathrm{Kn}=10$ is about $20 \%$ (with respect to the ideal case of $\phi=1$ ), whereas at $\mathrm{Kn}=$ 0.025 and $U_{\infty}=30 \mathrm{~m} / \mathrm{s}$ the corresponding reduction is only about $13.4 \%$. As the flow approaches the free molecular regime, rarefaction effects become predominant and there is a greater chance of molecular condensation at the interface due to the fact that the collision frequency decreases. 


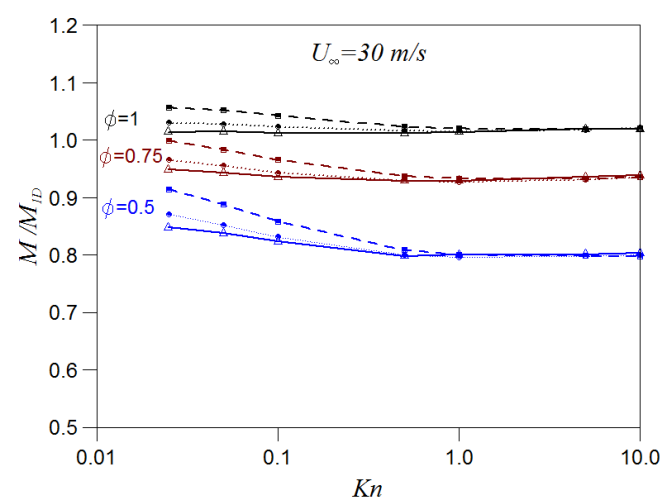

(a)

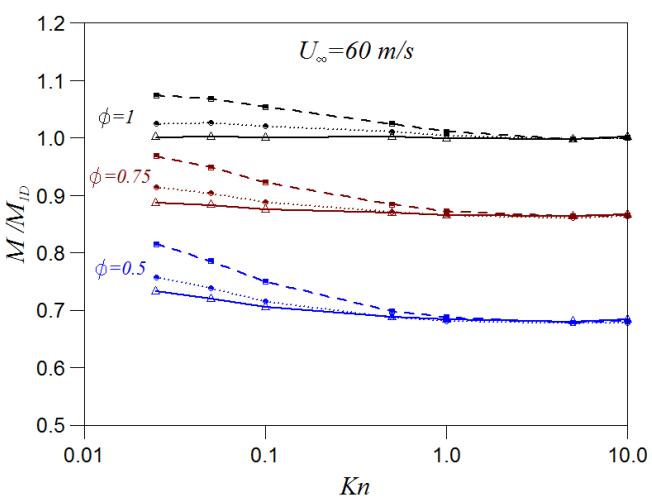

(b)

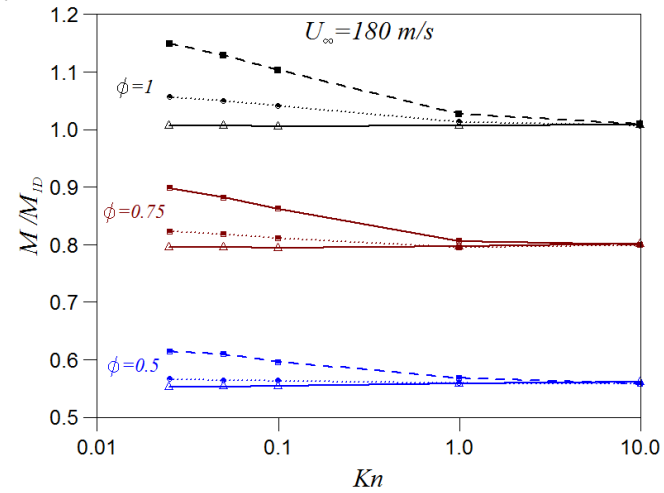

(c)

FIG. 12. Normalized mass flux versus the Knudsen number plotted for three selected free-stream velocities. Dashed lines represent the semicircle meniscus, $S_{3}$; dotted lines represent the curved meniscus, $S_{2}$; and solid lines represent the flat meniscus, $S_{1}$.

\section{Impact of the evaporation coefficient}

All the results presented so far have considered an evaporation coefficient of unity. Although $\sigma=1$ is considered generally to be a reasonable assumption, previous studies based on experimental [53,54], transition state theory [55], and molecular dynamics [56-58] studies suggest that it is possible for the evaporation coefficient to be significantly less than 1 under certain conditions, particularly for polyatomic gases like water, methanol, and refrigerants. It is also sensitive to interface conditions like temperature. It is therefore very important to study the dependence of evaporation coefficient on the performance of nanoporous membranes. To investigate the effect of the evaporation coefficient $\sigma$ on the evaporation rates, a selected fixed free-stream velocity of $U_{\infty}=60 \mathrm{~m} / \mathrm{s}$ has been considered for all cases in this part of the study. First, we study the impact of nonunity evaporation coefficients for evaporation from a planar surface in the one-dimensional limit. For this, the computed mass flux for different values is plotted together with its analytical solution [from Eq. (6)] in Fig 15. Excellent agreement can be noted between the computed and analytical results. The absolute value of mass flux clearly decreases with any reduction in the evaporation coefficient.

The impact of $\sigma$ on the evaporation rates for different meniscus shapes is investigated next. The computed mass flux as a function of $\sigma$ is shown in Figs. 16(a) and 16(b) for $\phi=1$ and two selected values of Knudsen number, i.e., $\mathrm{Kn}=0.1$ and $\mathrm{Kn}=10$. The mass flux in these plots has been normalized with the analytical mass flux, $M_{1 D, \sigma}$, corresponding to the evaporation coefficient under 


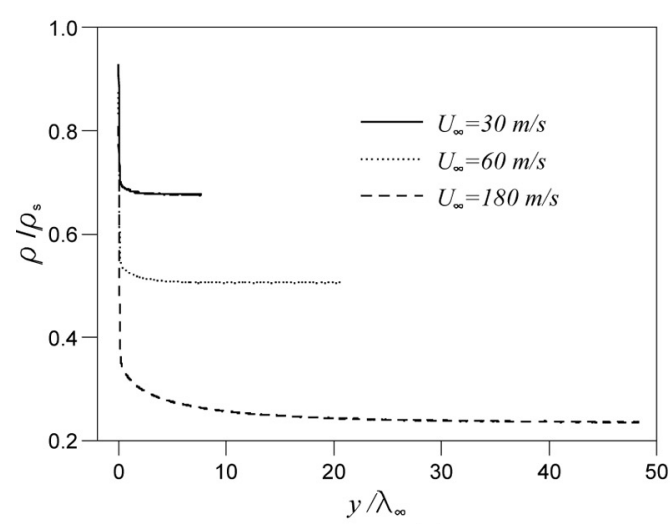

(a)

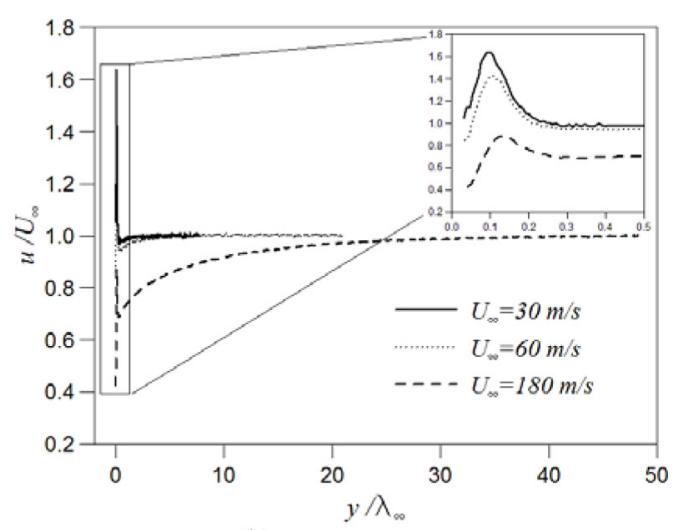

(b)

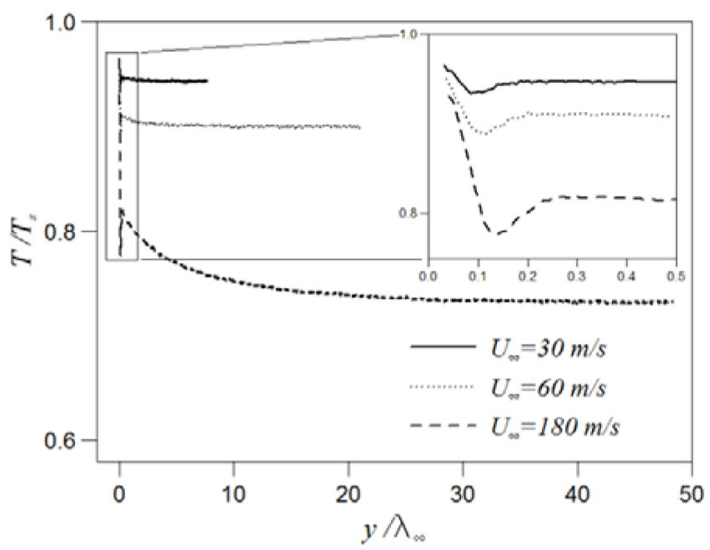

(c)

FIG. 13. Variation in (a) $\rho / \rho_{s}$, (b) $u / U_{\infty}$, and (c) $T / T_{s}$ plotted along the extent of the computational domain at $\mathrm{Kn}=10$ and three different free-stream velocities for the flat meniscus, $S_{1}$.

consideration. The absolute magnitude of mass flux is proportional to the evaporation coefficient, as expected for all meniscus cases (not shown). More interestingly, the curved meniscus cases attain a significantly higher mass flux relative to the flat meniscus case, $S_{1}$, particularly as $\sigma$ decreases. For instance, when $\sigma=0.1$, the semicircle meniscus, $S_{3}$, predicts about $40 \%$ higher mass flux than $S_{1}$. The reason for this can be attributed to the fact that all the molecules crossing the interface are lost (condensed) for $\sigma=1$. However, for $\sigma<1$, a fraction of the molecules (proportional to $1-\sigma$ ) crossing the interface get reemitted. This clearly makes a difference, as it can be noted that the variations in mass flux between the meniscus cases is minimal when $\sigma=1$; however, as $\sigma$ reduces, the mass flux becomes proportional to the interface surface (i.e., area per unit width). In particular, as $\sigma \rightarrow 0$, the ratio of mass flux predicted by the semicircle meniscus to that by the flat meniscus approaches $\pi / 2$ (the ratio of the semicircle meniscus to the flat meniscus).

The evaporation coefficient sensitivity study has also been done for porosity $\phi=0.75$, and the corresponding results are shown in Figs. 16(c) and 16(d). The trends are similar to those for $\phi=1$. The variations in mass flux between the meniscus cases are again relatively minimal when $\sigma=1$, for both Knudsen numbers (particularly for $\mathrm{Kn}=10$ ). However, as $\sigma$ reduces, the curved meniscus cases predict a larger mass flux as the evaporation rates becomes proportional to the interfacial surface. 


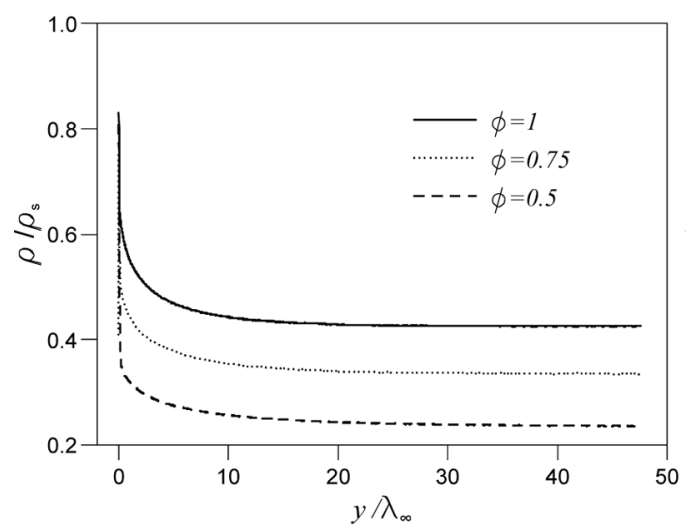

(a)

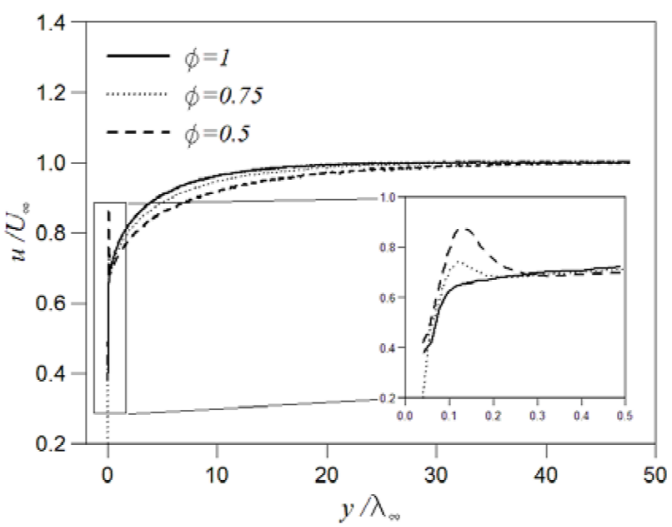

(b)

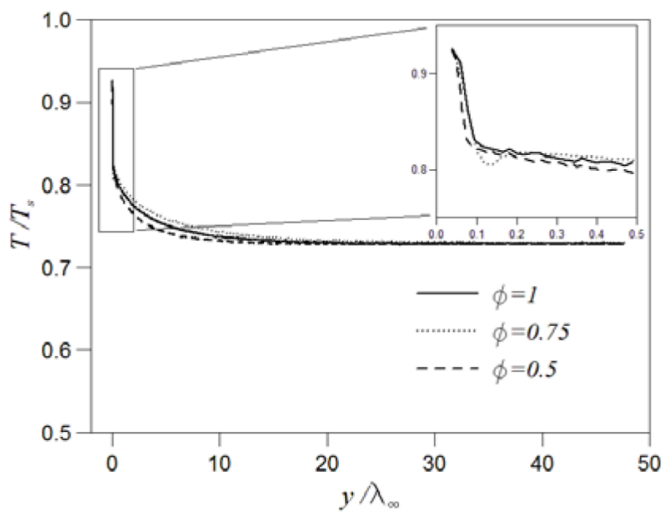

(c)

FIG. 14. Variation in (a) $\rho / \rho_{s}$, (b) $u / U_{\infty}$, and (c) $T / T_{s}$ plotted along the extent of the computational domain at $\mathrm{Kn}=10$ and $U_{\infty}=180 \mathrm{~m} / \mathrm{s}$, but at different porosities for the flat meniscus, $S_{1}$.

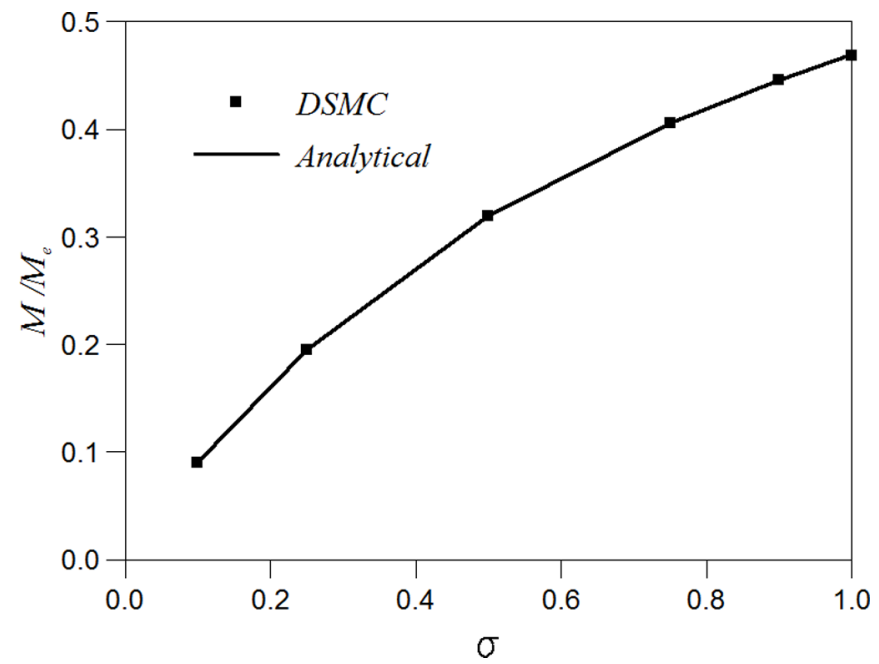

FIG. 15. Validation of computed mass flux as a function of the evaporation coefficient against the analytical solution for a fixed free-stream velocity, $U_{\infty}=60 \mathrm{~m} / \mathrm{s}$. 


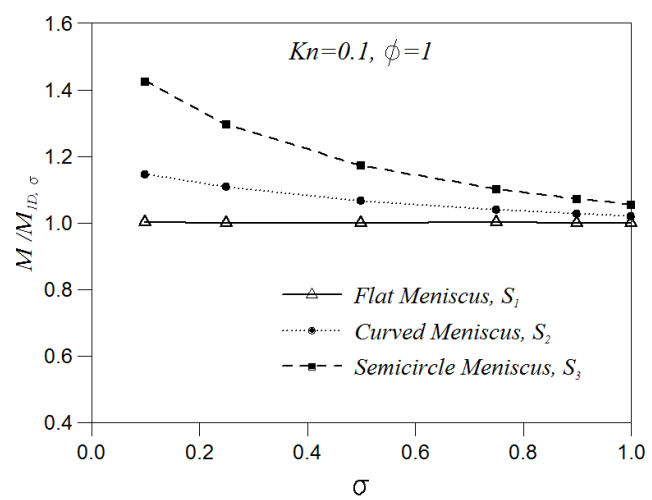

(a)

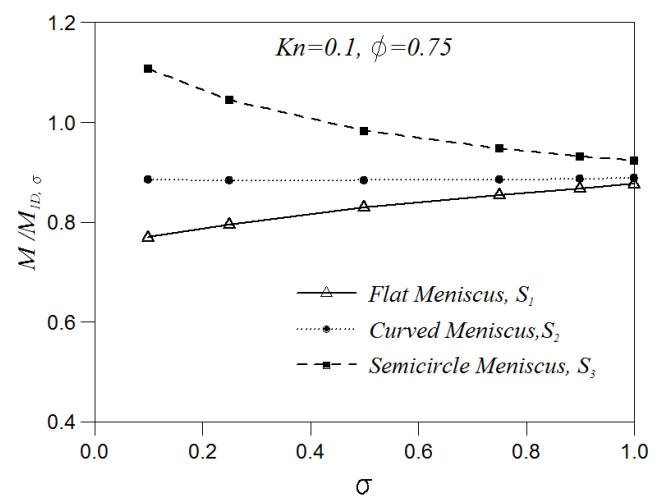

(c)

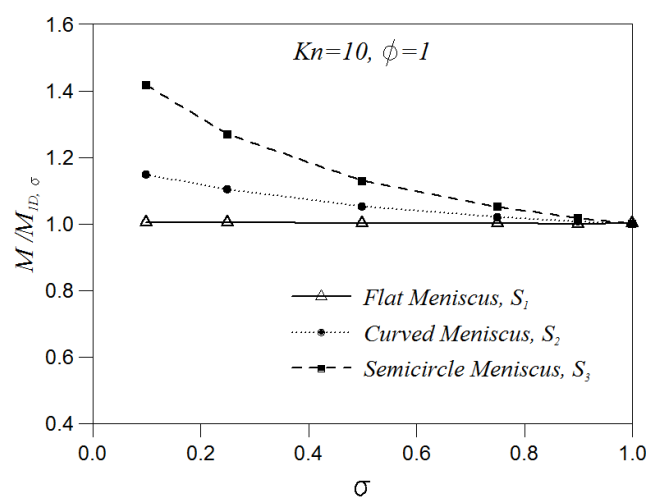

(b)

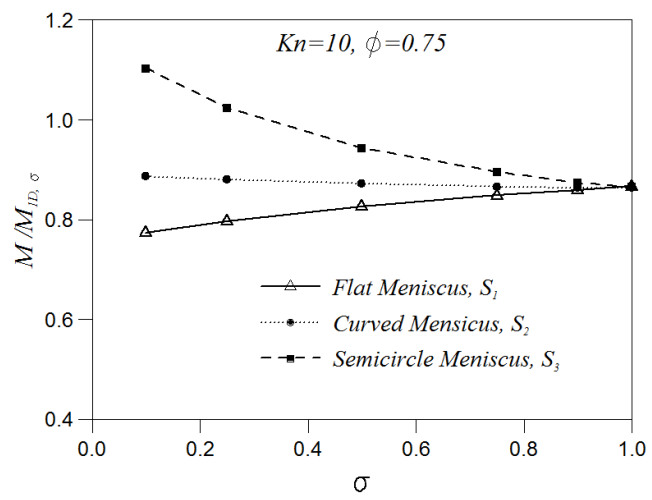

(d)

FIG. 16. Normalized mass flux as a function of the evaporation coefficient for different values of $\phi$ and $\mathrm{Kn}$, computed for a fixed free-stream velocity, $U_{\infty}=60 \mathrm{~m} / \mathrm{s}$.

\section{E. Receding meniscus cases}

So far, we have considered an ideal meniscus case, i.e., when the meniscus is pinned to the top of the pore. In practice, the meniscus could actually recede down the pore under a range of operating conditions, such as a higher pore surface temperature or heat flux. Therefore, receding meniscus cases need to be investigated to assess their influence on nanopore efficacy. Figure 17 illustrates a receding meniscus case, where $h$ denotes the distance by which the top of the meniscus is below the top of the nanopore.

Receding meniscus cases have been considered by studying three selected cases of $h$, i.e., $h=$ $L_{p} / 6, L_{p} / 2$, and $2 L_{p}$, and comparing their respective evaporation rates with the ideal pinned meniscus case, i.e., $h=0$. A fixed free-stream velocity, $U_{\infty}=60 \mathrm{~m} / \mathrm{s}$, is considered for all cases in this part of the study. Figure 18 shows the computed normalized flux as a function of $\mathrm{Kn}$ for different values of $h$, at two porosities, $\phi=1$ and $\phi=0.75$. It can be noted that as the meniscus recedes down the pore the evaporation rates reduce significantly. This can be attributed to the additional losses (vapor transport resistance) encountered, as the vapor travels up the pore before it eventually escapes. For instance, when the meniscus recedes by a distance of just $2 L_{p}$, the mass flux reduces by about $28 \%$ for $\mathrm{Kn}=10$ and $\phi=1$. The substantial reduction in evaporation rates is generally consistent with experimental findings [19]. More interestingly, the trend in the variation of evaporation rate with $\mathrm{Kn}$ alters significantly as the meniscus recedes down the pore. For small values of $h$ (i.e., $L_{p} / 6$ and $L_{p} / 2$ ), the trends in the variation of mass flux with $\mathrm{Kn}$ are similar to the pinned meniscus case. As the meniscus recedes down the pore, the behavior changes and is now noted to be similar to the 


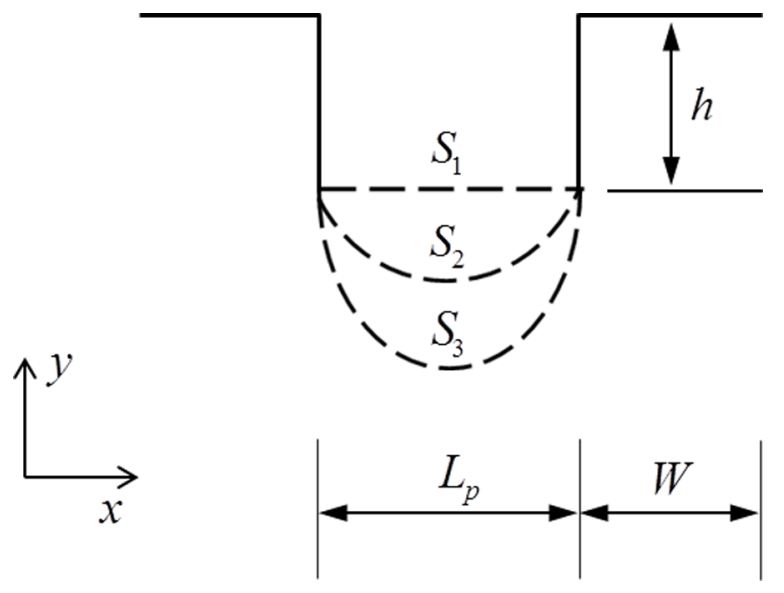

FIG. 17. Schematic of the receding meniscus case.

mass flux variation in Poiseuille flow. For $h=2 L_{p}$, the mass flux first decreases as Kn increases and then increases with a Knudsen number minimum in the transition regime. This behavior holds true for both cases of porosity considered.

We also examine the effect of the evaporation coefficient on the receding meniscus case. A selected evaporation coefficient of $\sigma=0.1$ and receding height $h=2 L_{p}$ are considered for this purpose. The evaporation rates for this receding case are plotted in Fig. 19. The mass flux in these plots has been normalized with the analytical mass flux, $M_{1 D}$, corresponding to $\sigma=0.1$. The apparent reduction in the magnitude of mass flux as $\sigma$ reduces can be clearly noted for $h=2 L_{p}$ [by comparing Fig. 19(a) against Fig. 18(a)]. More interestingly, at $\sigma=0.1$, the curved meniscus cases consistently produce a much higher mass flux when compared to the flat meniscus cases, for all degrees of flow rarefaction. Evidently, as $\sigma$ reduces, mass flux is consistently proportional to the interface surface, and this behavior holds true irrespective of the Knudsen number under consideration. Quantitatively, the ratio of mass flux predicted by the semicircle meniscus to that by the flat meniscus is noted to be about 1.4 for $\sigma=0.1$, which suggests that as $\sigma \rightarrow 0$ this ratio will again approach $\pi / 2$. Comparison of the receding case with the pinned case for $\sigma=0.1$ is also made

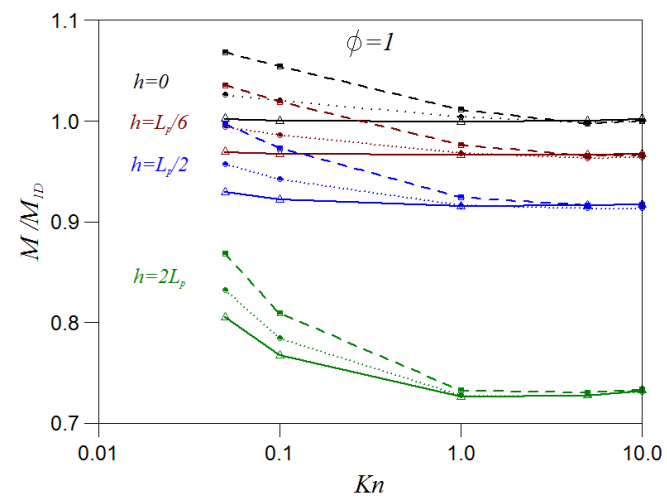

(a)

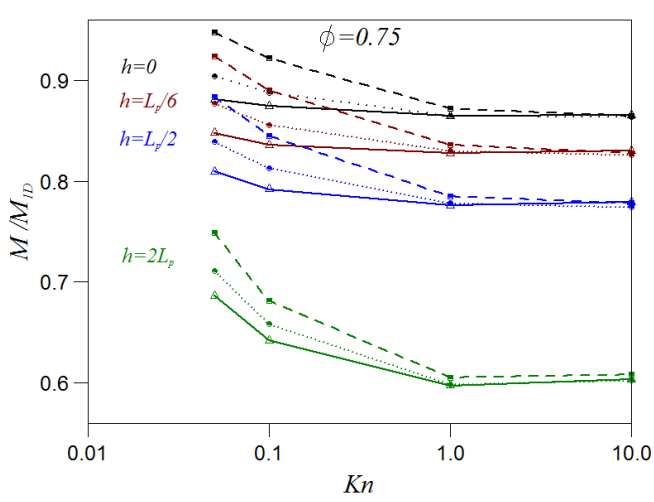

(b)

FIG. 18. Computed normalized mass flux as a function of Knudsen number for different values of $h$. The line types represent different meniscus shapes; i.e., solid lines denote the flat meniscus, $S_{1}$; dotted lines represent the curved meniscus, $S_{2}$; dashed lines represent the semicircle meniscus, $S_{3}$. 


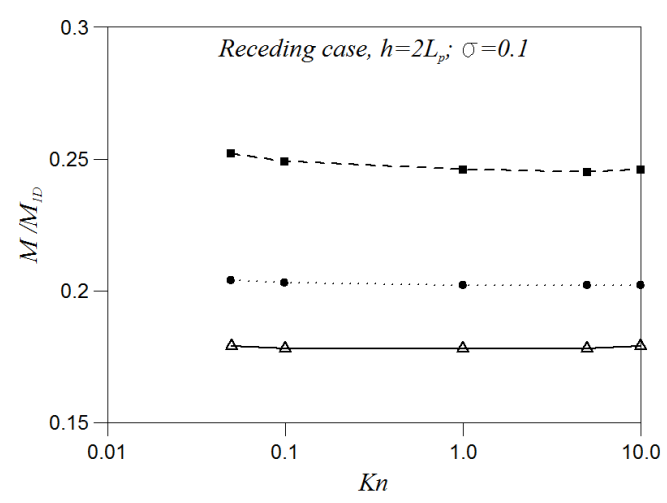

(a)

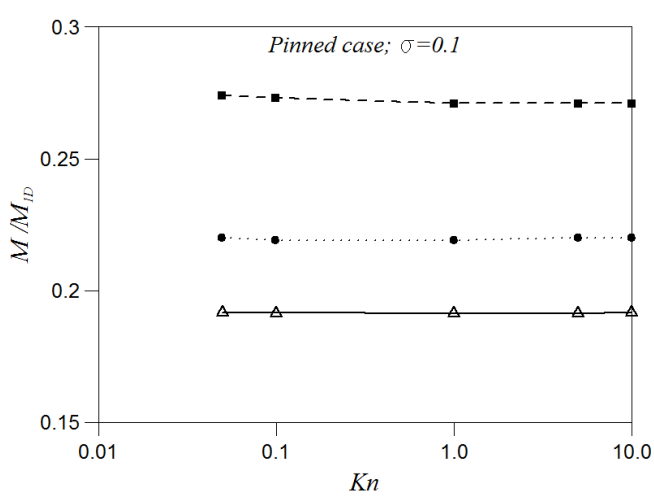

(b)

FIG. 19. Comparison of the computed mass flux between the receding case $\left(h=2 L_{p}\right)$ and the pinned case ( $h=0$ ) for $\sigma=0.1$ and $\phi=1$. The line types represent different meniscus shapes; i.e., solid lines denote the flat meniscus, $S_{1}$; dotted lines represent the curved meniscus, $S_{2}$; dashed lines represent the semicircle meniscus, $S_{3}$.

in Fig. 14. It can be noted that the trends in the variation of evaporation rates between the receding and pinned cases for $\sigma=0.1$ are similar. Additionally, unlike for an evaporation coefficient of unity, the magnitude of mass flux is relatively similar for both receding and pinned meniscus cases when $\sigma=0.1$, i.e., the relative mass flux reduction (losses) as the meniscus recedes down the pore becomes minimal for very low values of $\sigma$.

\section{CONCLUSIONS}

In the present study, the direct simulation Monte Carlo method has been used to investigate the gas dynamics associated with evaporation processes from a model nanoporous membrane cooling device. In particular, the evaporation from representative nanoporous meniscus shapes corresponding to different operating conditions has been investigated. Evaporation rates and the corresponding free-stream to interface density ratios and temperature jumps have been characterized as a function of a wide range of interface Knudsen numbers and free-stream Mach numbers. Simulations in this work have led to several insights into the nanoporous evaporation phenomena. The main findings of this work are as follows:

(1) The meniscus shape does not play a critical role, for relatively large Knudsen numbers (from the early transition to free molecular regime) and evaporation coefficient close to unity, as the curved menisci predict an evaporation rate similar to that of the flat meniscus case. This is due to the fact that a higher Knudsen number favors a relatively greater chance of condensation due to lower collision frequency near the interface. On the other hand, at lower Knudsen numbers (in the continuum and slip regimes), the meniscus shape plays a key role as the curved meniscus cases consistently produce a larger mass flux in comparison to a flat meniscus. This is particularly so at higher free-stream velocities, since a higher flow velocity near the interface (for the same Kn) reduces the backscattered fraction of molecules condensing at the interface.

(2) Small changes in porosity lead to substantial reductions in mass flux, particularly as the free-stream Mach number (or indirectly, the interface to free-stream density ratio) increases. This can be attributed to the combined effect of the decrease in the relative available evaporative interface surface as porosity decreases and the increased role of the gas expansion phenomenon as free-stream Mach number increases.

(3) The choice of evaporation coefficient has been found to have a significant effect on the evaporation rates. This is due to the relative importance of the molecular emission and reemission phenomenon at the interface varying with the evaporation coefficient. In particular, for low values 
of the evaporation coefficient, the curved meniscus cases attain a significantly higher mass flux irrespective of the interface Knudsen number conditions.

(4) The relative evaporation rates for the receding menisci drop significantly, since the vapor encounters additional losses as it travels up the pore. Additionally, it was found that the trend in the variation of evaporation rate with Knudsen number alters significantly with the location of the receding meniscus. Furthermore, an analysis of the impact of evaporation coefficient on the receding meniscus cases has revealed that the relative mass flux reduction as the meniscus recedes down the pore becomes minimal for very low values of evaporation coefficient.

In summary, this work has demonstrated that the efficacy of the nanoporous membrane needs to be carefully examined as it is ultimately determined by the interplay between a host of relevant parameters, including the meniscus shape, Knudsen number, porosity, and the evaporation coefficient. This work on nanoporous evaporation thus not only provides useful information for the design of novel nanoporous membrane-based cooling devices but also has relevance to related applications, such as sea water desalination using carbon nanotubes. Finally, future work will consider a wider range of parameter space to cover additional relevant combinations of porosity, evaporation coefficient, and receding menisci that have not been considered in this study. In particular, we will explore in the near future the feasibility of an effective one-dimensional approach to predict the mass flux and jump relations across the Knudsen layer for arbitrary nanoporous configurations, through specification of an appropriate effective evaporation coefficient.

\section{ACKNOWLEDGMENTS}

This work was supported through the United Kingdom Engineering and Physical Sciences Research Council (EPSRC) under Grant No. EP/N016602/1, "Nano-Engineered Flow Technologies: Simulation for Design across Scale and Phase." J.S. acknowledges additional support from EPSRC Grants No. EP/P020887/1 and No. EP/P031684/1. The simulations in this work were carried out on the UKs National Supercomputing service, ARCHER; resources were awarded through an ARCHER Resource Allocation Panel award. The authors also thank the reviewers for their useful suggestions.

[1] X. Liu, M. H. Hu, C. G. Caneau, R. Bhat, and C.-E. Zah, Thermal management strategies for high power semiconductor pump lasers, IEEE Trans. Compon. Packag. Manuf. Technol. 29, 268 (2006).

[2] E. Pop, Energy dissipation and transport in nanoscale devices, Nano Res. 3, 147 (2010).

[3] A. Bartolini, M. Cacciari, A. Tilli, and L. Benini, Thermal and energy management of high-performance multicores: Distributed and self-calibrating model-predictive controller, IEEE Trans. Parallel Distrib. Syst. 24, 170 (2013).

[4] B. H. Liou, C. M. Chen, R. H. Horng, Y. C. Chiang, and D. S. Wu, Improvement of thermal management of high-power GaN-based light-emitting diodes, Microelectron. Reliab. 52, 861 (2012).

[5] R. S. Pengelly, S. M. Wood, J. W. Milligan, S. T. Sheppard, and W. L. Pribble, A review of GaN on SiC high electron mobility power transistors and MMICs, IEEE Trans. Microwave Theory Tech. 60, 1764 (2012).

[6] S. You, J. Kim, and K. Kim, Effect of nanoparticles on critical heat flux of water in pool boiling heat transfer, Appl. Phys. Lett. 83, 3374 (2003).

[7] R. Chen, M.-C. Lu, V. Srinivasan, Z. Wang, H. H. Cho, and A. Majumdar, Nanowires for enhanced boiling heat transfer, Nano Lett. 9, 548 (2009).

[8] S. G. Kandlikar, Fundamental issues related to flow boiling in minichannels and microchannels, Exp. Therm. Fluid Sci. 26, 389 (2002).

[9] A. Kosar, C. J. Kuo, and Y. Peles, Suppression of boiling flow oscillations in parallel microchannels by inlet restrictors, J. Heat Transfer 128, 251 (2006). 
[10] L. Lin and R. Ponnappan, Heat transfer characteristics of spray cooling in a closed loop, Int. J. Heat Mass Transfer 46, 3737 (2003).

[11] Z. Lu, T. R. Salamon, S. Narayanan, K. R. Bagnall, D. F. Hanks, D. S. Antao, B. Barabadi, J. Sircar, M. E. Simon, and E. N. Wang, Design and modeling of membrane-based evaporative cooling devices for thermal management of high heat fluxes, IEEE Trans. Compon. Packag. Manuf. Technol. 6, 1056 (2016).

[12] J. L. Plawsky, A. G. Fedorov, S. V. Garimella, H. B. Ma, S. C. Maroo, L. Chen, and Y. Nam, Nano- and microstructures for thin-film evaporation - A review, Nanoscale Microscale Thermophys. Eng. 18, 251 (2014).

[13] S. Adera, D. Antao, R. Raj, and E. N. Wang, Design of micropillar wicks for thin-film evaporation, Int. J. Heat Mass Transf. 101, 280 (2016).

[14] C. Ding, G. Soni, P. Bozorgi, B. D. Piorek, C. D. Meinhart, and N. C. MacDonald, A flat heat pipe architecture based on nanostructured titania, J. Microelectromech. Syst. 19, 878 (2010).

[15] L. Swanson and G. Herdt, Model of the evaporating meniscus in a capillary tube, J. Heat Transf. 114, 434 (1992).

[16] H. Wang, S. V. Garimella, and J. Y. Murthy, Characteristics of an evaporating thin film in a microchannel, Int. J. Heat Mass Transf. 50, 3933 (2007).

[17] S. Narayanan, A. G. Fedorov, and Y. K. Joshi, Interfacial transport of evaporating water confined in nanopores, Langmuir 27, 10666 (2011).

[18] Z. Lu, S. Narayanan, and E. N. Wang, Modeling of evaporation from nanopores with nonequilibrium and nonlocal effects, Langmuir 31, 9817 (2015).

[19] K. L. Wilke, B. Barabadi, Z. Lu, T. Zhang, and E. N. Wang, Parametric study of thin film evaporation from nanoporous membranes, Appl. Phys. Lett. 111, 171603 (2017).

[20] T. Humplik, J. Lee, S. O’Hern, B. Fellman, M. Baig, S. Hassan, M. Atieh, F. Rahman, T. Laoui, and R. Karnik, Nanostructured materials for water desalination, Nanotechnol. 22, 292001 (2011).

[21] J. Lee, T. Laoui, and R. Karnik, Nanofluidic transport governed by the liquid/vapor interface, Nat. Nanotechnol. 9, 317 (2014).

[22] D. L. Gin and R. D. Noble, Designing the next generation of chemical separation membranes, Science 332, 674, (2011).

[23] R. H. Rand, Fluid mechanics of green plants, Annu. Rev. Fluid Mech 15, 29 (1983).

[24] T. Ytrehus, Molecular-flow effects in evaporation and condensation at interfaces, Multiphase Sci. Technol. 9, 205 (1997).

[25] H. Hertz, Uber die Verdunstung der Flüssigkeiten, insbesondere des Quecksilbers, im luftleeren Raume, Ann. Phys. (Leipzig) 253, 177 (1882).

[26] M. Knudsen, Die maximale Verdampfungsgeschwindigkeit des Quecksilbers, Ann. Phys. (Leipzig) 352, 697 (1915).

[27] R. W. Schrage, A Theoretical Study of Interphase Mass Transfer (Columbia University Press, New York, 1953).

[28] D. Labuntsov and A. Kryukov, Analysis of intensive evaporation and condensation, Int. J. Heat Mass Transf. 22, 989 (1979).

[29] S. M. Yen, Numerical solutions of non-linear kinetic equations for a one-dimensional evaporationcondensation problem, Comput. Fluids 1, 367 (1973).

[30] Y. Sone, K. Aoki, H. Sugimoto, and T. Yamada, Steady evaporation and condensation on a plane condensed phase, Theor. Appl. Mech. (Bulgaria) 19, 89 (1988).

[31] K. Aoki, Y. Sone, and T. Yamada, Numerical analysis of gas flows condensing on its plane condensed phase on the basis of kinetic theory, Phys. Fluids A 2, 1867 (1990).

[32] G. A. Bird, Molecular Gas Dynamics and the Direct Simulation of Gas Flows (Clarendon, Oxford, 1998).

[33] M. Murakami, and K. Oshima, Kinetic approach to the transient evaporation and condensation problem, in Rarefied Gas Dynamics, edited by M. Becker and M. Fiebig (DFVLR Press, Portz-Wahn, 1974), p. F6.

[34] M. N. Kogan and A. A. Abramov, Direct simulation solution of the strong evaporation and condensation problem, in Rarefied Gas Dynamics, edited byA. E. Beylich (VCH, Weinheim, 1990), p. 1251.

[35] Y. Sone, Kinetic theoretical studies of the half-space problem of evaporation and condensation, Transp. Theory Stat. Phys. 29, 227 (2000). 
[36] C. Cercignani, Strong evaporation of a polyatomic gas, in Rarefied Gas Dynamics, Proceedings of the 12th International Rarefied Gas Dynamics symposium, edited by S. S. Fisher (AIAA, Reston, VA, 1981), pp. 305-320.

[37] A. Frezzotti, Kinetic theory description of the evaporation of multi-component substances, in 20th International Symposium on Rarefied Gas Dynamics (Peking University Press, Beijing, 1997), p. 837.

[38] A. Frezzotti, A numerical investigation of the steady evaporation of a polyatomic gas, Eur. J. Mech. B: Fluids 26, 93 (2007).

[39] Y. Sone and H. Sugimoto, Kinetic theory analysis of steady evaporating flows from a spherical condensed phase into vacuum, Phys. Fluids A: Fluid Dyn. 5, 1491 (1993).

[40] R. H. Edwards and R. L. Collins, Evaporation from a spherical source into a vacuum, in Rarefied Gas Dynamics, edited by L. Trilling and H. Y. Wachmann (Academic, New York, 1969), p. 1489.

[41] H. Sugimoto and Y. Sone, Numerical analysis of steady flow of a gas evaporating from its cylindrical condensed phase on the basis of kinetic theory, Phys. Fluids A 4, 419 (1992).

[42] B. Derjaguin, N. Churaev, and V. Muller, Wetting Films, Surface Forces (Springer, Berlin, 1987).

[43] A. A. Avdeev and Y. B. Zudin, Kinetic analysis of intensive evaporation (method of reverse balances), High Temp. 50, 527 (2012).

[44] J. W. Rose, Accurate approximate equations for intensive subsonic evaporation, Int. J. Heat Mass Transf. 43, 3869 (2000).

[45] J. Lee and R. Karnik, Desalination of water by vapor-phase transport through hydrophobic nanopores, J. Appl. Phys. 108, 044315 (2010).

[46] A. Berman, Free molecule transmission probabilities, J. Appl. Phys. 36, 3356 (1965).

[47] Z. Lu, I. Kinefuchi, K. Wilke, G. Vaartstra, and E. Wang, A unified relationship for evaporation kinetics at low Mach numbers, Nat Commun. 10, 2368 (2019).

[48] A. Frezzotti, Boundary conditions at the vapor-liquid interface, Phys. Fluids 23, 030609 (2011).

[49] S. J. Plimpton and M. A. Gallis, SPARTA Direct Simulation Monte Carlo (DSMC) Simulator, http://sparta.sandia.gov, 2015.

[50] M. A. Gallis, J. R. Torczynski, S. J. Plimpton, D. J. Rader, and T. Koehler, Direct simulation Monte Carlo: The quest for speed, in Proceedings of the 29th International Symposium on Rarefied Gas Dynamics, edited by J. Fan, AIP Conf. Proc. No. 1628 (AIP, New York, 2014), p. 27.

[51] M. A. Gallis, T. P. Koehler, J. R. Torczynski, and S. J. Plimpton, Direct simulation Monte Carlo investigation of the Rayleigh-Taylor instability, Phys. Rev. Fluids 1, 043403 (2016).

[52] B. John, D. A. Lockerby, A. Patronis, and D. R. Emerson, Simulation of the head-disk interface gap using a hybrid multi-scale method, Microfluid. Nanofluid. 22, 106 (2018).

[53] M. Maerefat, S. Fujikawa, T. Akamatsu, and T. Mizutani, An experimental study of non-equilibrium vapor condensation in a shock-tube, Exp Fluids 7, 513 (1989).

[54] T. Tsuruta, T. Masuoka, and Y. Kato, Estimation of condensation coefficient from dropwise condensation heat transfer, Thermal Sci. Eng. 2, 98 (1994).

[55] S. Fujikawa and M. Maerefat, A study of the molecular mechanism of vapor condensation, JSME Int. J. 33, 634 (1990).

[56] M. Matsumoto, K. Yasuoka, and Y. Kataoka, Molecular simulation of evaporation and condensation, Fluid Phase Equilib. 104, 431 (1995).

[57] M. Matsumoto, K. Yasuoka, and Y. Kataoka, Evaporation and condensation at a liquid surface: 2. Methanol, J. Chem. Phys. 101, 7912 (1994).

[58] T. Tsuruta and G. Nagayama, A microscopic formulation of condensation coefficient and interface transport phenomena, Energy 30, 795 (2005). 